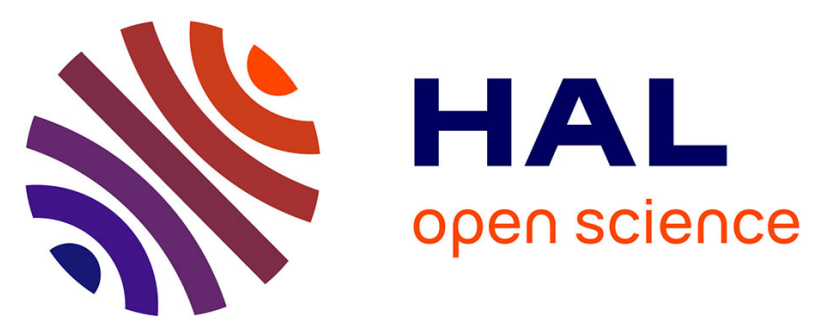

\title{
Impinging cross-shaped submerged jet on a flat plate: a comparison of plane and hemispherical orifice nozzles
}

\author{
Kodjovi Sodjavi, Brice Montagné, Pierre Bragança, Amina Meslem, Florin
}

Bode, Magdalena Kristiawan

\section{- To cite this version:}

Kodjovi Sodjavi, Brice Montagné, Pierre Bragança, Amina Meslem, Florin Bode, et al.. Impinging cross-shaped submerged jet on a flat plate: a comparison of plane and hemispherical orifice nozzles. Meccanica, 2015, 50 (12), pp.2927-2947. 10.1007/s11012-015-0181-5 . hal-01165569

HAL Id: hal-01165569

https://hal-univ-rennes1.archives-ouvertes.fr/hal-01165569

Submitted on 19 Jun 2015

HAL is a multi-disciplinary open access archive for the deposit and dissemination of scientific research documents, whether they are published or not. The documents may come from teaching and research institutions in France or abroad, or from public or private research centers.
L'archive ouverte pluridisciplinaire HAL, est destinée au dépôt et à la diffusion de documents scientifiques de niveau recherche, publiés ou non, émanant des établissements d'enseignement et de recherche français ou étrangers, des laboratoires publics ou privés. 


\title{
Impinging Cross-Shaped submerged Jet on a Flat Plate: a Comparison of Plane and Hemispherical Orifice Nozzles
}

\author{
Kodjovi Sodjavi ${ }^{\mathrm{a}}$, Brice Montagné ${ }^{\mathrm{a}}$, Pierre Bragança ${ }^{\mathrm{a}}$, Amina Meslem $^{\mathrm{b}}{ }^{*}$, Florin Bode $^{\mathrm{c}}$, \\ Magdalena Kristiawan ${ }^{\mathrm{d}}$,
}

\footnotetext{
a LaSIE, University of La Rochelle, Pôle Sciences et Technologie, Avenue Michel Crépeau, 17042 La Rochelle, France

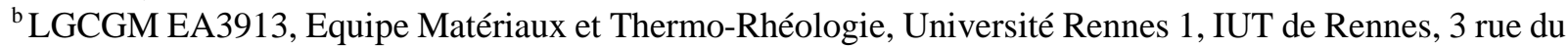
Clos Courtel, BP 90422, 35704 Rennes Cedex 7, France, amina.meslem@ univ-rennes1.fr,* corresponding author

${ }^{c}$ Technical University of Cluj-Napoca, Faculty of Mechanical Engineering, Avenue Muncii 103-105, ClujNapoca, Romania

${ }^{\mathrm{d}}$ Institut National de la Recherche Agronomique, BIA, BP 71627, 44316 Nantes, France
}

\begin{abstract}
It is well known that the transfer of heat, mass and momentum to a wall by an impinging jet is partially linked to the way in which jet generation is realized. The organization of the vortices at the jet exit depends on the upstream conditions and on the geometry of the nozzle. Particle Image Velocimetry (PIV) and electrodiffusion techniques were used to investigate the characteristics of different impinging jets and the resulting wall shear rates and mass transfer. Two Cross-Shaped orifice jets, one produced by a plane orifice nozzle (i.e. a Cross-Shaped Orifice made on a flat Plate, $\mathrm{CO} / \mathrm{P}$ ) and the second by a hemispherical orifice nozzle (i.e. a Cross-Shaped Orifice made on a Hemisphere, $\mathrm{CO} / \mathrm{H}$ ), were compared to a reference round jet produced by a convergent nozzle. The distance between the jet exit and the target wall was equal to two nozzle equivalent diameters $\left(D_{e}\right)$, based on the free orifice area. The Reynolds number, based on $D_{e}$ and on the exit bulk-velocity, was 5620 for each flow. PIV measurements give an overall view of the flow characteristics in their free and wall regions. The switching-over phenomena observed in the CO/P nozzle case, and already described in the literature with similar nozzles, did not occur in the jet from the $\mathrm{CO} / \mathrm{H}$ nozzle. Electrodiffusion measurements showed differences in the shape and level of radial distribution of the wall shear rates and mass transfer. One of the most important observations is the large difference in wall shear stress between the three jets. For the same exit bulk-velocity, the maximum wall shear rate in the $\mathrm{CO} / \mathrm{P}$ and $\mathrm{CO} / \mathrm{H}$ nozzle jets was almost two and three times higher, respectively, than that of the reference convergent jet. This higher wall shear rate is accompanied
\end{abstract}


by higher mass transfer rate. It is demonstrated that the Cross-Shaped orifices enhance the mass transfer not only locally but also globally.

Keywords: impinging jet, lobed jet, wall-shear rate, mass transfer, flow dynamics

\section{List of symbols}

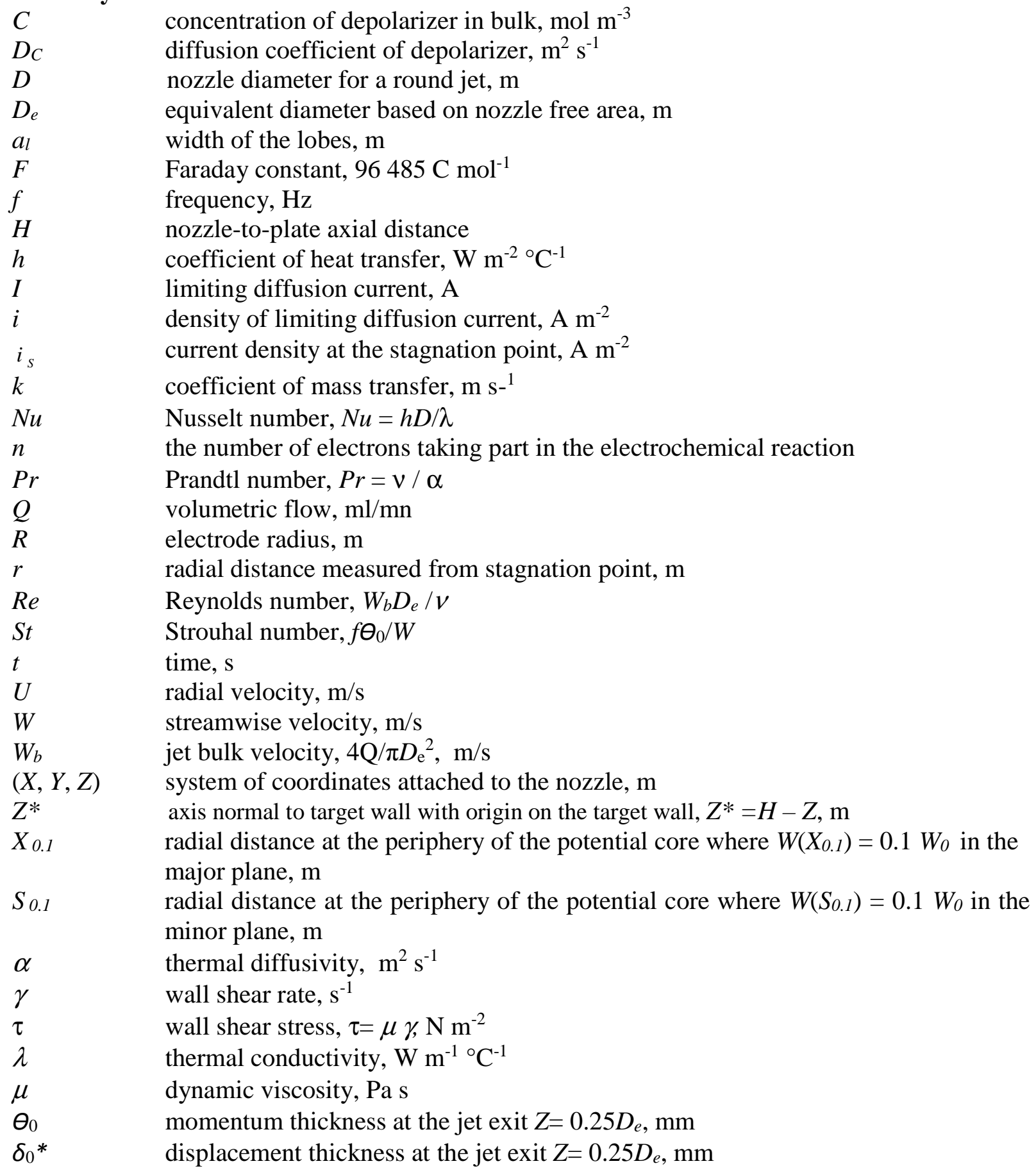




$\begin{array}{ll}v & \text { kinematic viscosity, } \mathrm{m}^{2} \mathrm{~s}^{-1} \\ \omega & \text { vorticity component, } \mathrm{s}^{-1} \\ & \text { Subscripts } \\ L & \text { Lévêque solution } \\ 0 & \text { jet value at the center of the nozzle and at } Z=0.25 D_{e} \\ C & \text { jet centerline value } \\ * & \text { axis taking its origin on the target wall }\end{array}$

\section{Introduction}

In the field of fundamental and applied research on turbulent jets, it was observed that the characteristics of initial shear layers and of the subsequent vortices which are formed in the near field of the jet are directly related to the inlet conditions governed by an intentional and/or an accidental excitation of the shear layer [1-6]. Active [7-9] or passive [10-13] alteration of the initial conditions causes considerable modifications of the large-scale vortices in both their shape and convection velocities. These techniques are commonly used to improve jet self-induction, which is useful in mixing processes such as those occurring in combustion chambers [6]. The influence of the initial conditions on jet dynamics is attributed to the underlying structure of turbulent motions that is carried from the jet inlet throughout the flow field. Thorough experiments, beginning with the contributions of Gardon et al. [14-16], were performed on heat/mass transfer using impinging jets, and an early observation was made about the importance of jet turbulence in heat transfer processes [15]. It was shown that some seemingly anomalous heat-transfer phenomena can be explained as effects of the turbulence occurring in jets. Turbulence is generated by the jet itself and by possible external disturbances and varies significantly with the nozzle shape, the upstream conditions and the position within the jet. One decade after the observation of Gardon and Akrifat [15], Popiel and Boguslawski [17] claimed that nozzle exit configuration was the most important factor affecting the stagnation point heat transfer. Despite these first very significant indications, there are only a few studies dedicated to heat/mass transfer enhancement using active [18-21] or passive jet control [22-26].

Gao et al. [24] were the first authors to introduce passive control of an impinging jet for heat transfer enhancement using vortex generators (triangular tabs). Heat transfer measurements were performed for impinging round pipe jets with a Reynolds number of 23000 and nozzle-to plate distances ranging from $1 D$ to $10 D$ where, $D$ is the nozzle diameter for a round jet. The authors found that the addition of triangular tabs at the pipe exit had a significant effect on the heat transfer 
produced by an impinging jet: the local heat transfer enhancement was in excess of $25 \%$. More recently, Herrero and Buchlin [27] tried to improve heat transfer using impinging lobed jets from straight pipe lobed nozzles. For different nozzle to plate distances, the authors compared the performance of their three-lobe and four-lobe nozzles to the square and rectangular nozzles of Gulati et al. [28] at a Reynolds number of 15000 . Even if the stagnation point Nusselt number of the three-lobe nozzle for $H / D_{e}=1$ was $10 \%$ higher than that of the other nozzles, the heat transfer enhancement was essentially similar to that obtained by Gulati et al. using a rectangular jet. According to Sang-Joon et al. [22], heat transfer enhancement in an asymmetric jet correlates with an increase in the entrainment rate. Lobed jets are known to significantly increase ambient fluid entrainment [11, 29-31], except when the lobed nozzle is a straight tube [30], as it was the case in Herrero and Buchlin's study [27].

As indicated above, a lot of studies have focused on the kinematic and the heat transfer behaviour of the impinging jets, while the local mass transfer process in such flow have received less attention. The available few studies on mass transfer in impinging jets have mainly used the electrochemical limiting diffusion current technique. Vallis et al. [32], Kataoka et al. [33] and Chin and Tsang [34] used this method for the local mass transfer measurement from a round impinging jet. Overall, there is a general consensus that the mass transfer is an increasing function of the Reynolds numbers.

The literature reveals that for high Reynolds numbers $(R e \geq 5000)$ and low nozzle-to-wall distances $(H / D<4)$, two peaks are present on the radial distribution of local Nusselt numbers produced by a circular impinging jet. The first peak corresponds to the maximum heat transfer rates and appears at the vicinity of the lateral edge of the nozzle. In some investigations the location of the first peak for $H / D<4$ was observed from $r=0.5 D$ to $r=0.7 D$ [20,23, 26, 35]. This peak was attributed to the high turbulence intensity at the nozzle edge and to a direct impingement of large toroidal Kelvin-Helmholtz $(\mathrm{K}-\mathrm{H})$ vortices originating in the mixing region. The secondary peak occurs at the radial distance from the stagnation point, from $1.2 D$ to $2.5 D[20,23,26,36]$. The second peak was either attributed to the transition from a laminar to a turbulent boundary layer in the wall jet region [16] or to the unsteady separation of the induced secondary vortices that form near the wall 
under primary $\mathrm{K}-\mathrm{H}$ vortices [37]. With the increase of Reynolds number, the location of the secondary peak moves outwards from the stagnation point and the peak height increases [23]. The distribution of Nusselt numbers $(\mathrm{Nu})$ generated by impinging jets has been characterized in numerous studies, but only a few investigations were devoted to the analysis of the corresponding wall shear rate $(\gamma)$ distribution $[33,35,38,39]$. Available data of $N u$ and $\gamma$ were compared and revealed similarities in their distribution, on the number of peaks and their radial location. For a high Reynolds number $(R e=41600)$ and a nozzle-to-wall distance of $H<4 D$, two peaks were found in $\gamma$ (or in wall shear stress $\tau=\mu . \gamma$ ) distribution [38]. As for the $N u$ - distribution [20, 23, $26,35]$, the first peak in $\gamma$-distribution appeared at a distance from the stagnation point ranging from $r=0.56 D$ to $r=0.74 D$, while the second peak was located at $r=1.9 D[38,40]$. The fact that peak-locations in $\gamma$-distribution closely match with those of $\mathrm{Nu}$-distribution suggests that the wall shear stress and the local heat/mass transfer are closely linked. This possibility is strengthened by the striking similarity between the instantaneous Nusselt number and the skin-friction coefficient observed a little further from the stagnation point in the LES simulation of Hadziabdic and Hanjalic [37].

The connection of the heat/mass transfer phenomena with the large-scale structures which develop in the free jet region or with the subsequent flow dynamics in the stagnation and the wall jet regions is now recognized [26, 37, 41, 42]. Therefore, the control of large-scale structures in impinging jets is a key element in the strategy of heat/mass transfer optimization and control. Passive control based on nozzle geometry modification is particularly attractive because it can easily be implemented for industrial applications.

Our previous investigations of free lobed jet dynamics and their mixing performance [30,43], along with the correlation that is mentioned in the literature between the mixing and heat/mass transfer phenomenon when the jet impinges a wall $[24,26]$, led us to focus our attention on the effect of lobed nozzle geometry on the transfer phenomena at the impinged wall.

This paper presents the first results of an optimization approach of injection geometry vis-à-vis wall shear stress and mass transfer enhancement resulting from jet impingement. Jet injection was performed using a cross-shaped orifice perforated on either a flat or hemispherical surface.

It has already been shown in our previous investigation of an impinging jet that a jet from a crossshaped orifice nozzle significantly increases the wall shear rate and stagnation mass transfer, 
compared to a reference jet from a convergent nozzle [44]. The hemispherical surface with a crossshaped orifice considered in the present study was designed to increase the stretching of the shearlayer at the jet exit in order to generate efficient jet dynamics in terms of wall skin friction and mass transfer. To ensure continuity with the previous investigation [44], we chose a similar jet Reynolds number (5620), and we used the two nozzles already studied (convergent and crossorifice cut on a flat plate), to which we added an innovative nozzle, i.e. the hemispherical crossorifice nozzle. Contrary to the previous investigation, where the nozzle-to-wall distance was varied in a range of $2 D_{e}-5 D_{e}\left(D_{e}\right.$ is the equivalent diameter based on the nozzle free area), this distance was kept constant, i.e. $H=2 D_{e}$ (with $D_{e}=7.8 \mathrm{~mm}$ for each nozzle); it corresponds to approximately the first half of the potential core length of a round free jet [45]. The Kelvin-Helmholtz toroidal vortices are well formed at this distance and are still well defined at the target wall. In the free lobed jets, the vortex mechanisms are very complex immediately after the nozzle exit [30, 31, 43, 46]. In this type of jet, the core length is reduced but is still greater than $2 D_{e}$. Thus with $H=2 D_{e}$, the wall is inside the potential core region for all the jets considered.

The present study aims to investigate the effects of the nozzle exit configuration on the flow features and on the resulting shear stress and mass transfer at the target wall.

In all the studied cases, the volumetric flow rate was conserved, and this choice is related to the aimed Heating, Ventilation and Air Conditioning (HVAC) application, and specifically to the Personalized Ventilation aspect [47]. For others applications where the energy conservation is required, further investigations should be conducted at the same power input.

The wall shear stress and the local mass transfer distributions were measured using electrodiffusion technique (ED), the same method we used before [44]. Phares et al. [39] made a critical survey of different techniques used for the measurement of wall shear stress and concluded that ED provided the greatest accuracy of any indirect method. To our knowledge, Kataoka et al. [35] were the first to introduce this technique for the measurement of wall skin friction generated by an impinging jet. While this method provides information on the gradient of the velocity in the vicinity of the wall, Particle Image Velocimetry (PIV) is used to measure the whole velocity field. Both techniques are complementary since PIV fails in the vicinity of the wall due to laser scattering by the solid surface. In our previous study [44], only the stagnation mass transfer has been provided and has been 
deduced from the slope of the radial wall shear rate distribution. In the present study, the radial mass transfer distribution along with global mass transfer are measured directly using the ED method. Furthermore, PIV measurements are performed in the considered flows, which was not the case in our previous study [44]. Hence a deeper analysis of mass transfer mechanisms in impinging lobed jets as a function of their kinematic is proposed.

The following was structured around two main sections. In Section 2 we outline the procedures employed. Sub-section 2.1 describes the experimental set-up and nozzle geometries, Sub-section 2.2 details the parameters of the PIV measurements and Sub-section 2.3 describes the ED measurement technique. Section 3 is dedicated to an analysis of the results. Sub-sections 3.1 and 3.2 discuss the features governing jet flow at the jet near field (Sub-section 3.1) and downstream (Sub-section 3.2), respectively, in the both the free and wall jet regions. Finally, Sub-section 3.3 analyzes the wall-shear rates and the mass transfer which are linked to the jet features before impingement.

\section{Experimental apparatus and methods}

\subsection{Jet flow generation}

The experiments were conducted in a liquid submerged jet impinging orthogonally onto a flat plate. A schematic diagram of jet generation in a reservoir is shown in Fig.1 a. A gear pump (from Ismatec with a GJ-N23 head) draws the liquid from a reservoir and delivers it to a nozzle. The liquid jet from the nozzle impinges a circular target disc equipped with six electrodes (Fig.1 b) which serve as probes for electrodiffusion (ED) measurements (see Sub-section 2.3 below). The temperature of the liquid is controlled by a cooling coil within $\pm 0.2^{\circ} \mathrm{C}$. The nozzle is screwed to a $200 \mathrm{~mm}$ long stainless steel tube with inner and outer diameters of 15 and $20 \mathrm{~mm}$, respectively. A honeycomb was manufactured by drilling 17 holes with a diameter of $2 \mathrm{~mm}$ into a $7 \mathrm{~mm}$ thick PVC disc which was then fitted into the tube inlet. The nozzle assembly was fixed to a holder that allowed vertical movement for accurate alignment of the nozzle axis with the centre of the electrodes. The reservoir was placed on a sliding compound table (Proxxon KT 150) which allowed movement in the axial and transverse directions relative to the nozzle, with a precision of $0.05 \mathrm{~mm}$. 
The target (Fig.1 b) was manufactured of a Plexiglas disc with a diameter of $100 \mathrm{~mm}$ and a thickness of $17 \mathrm{~mm}$ by first drilling holes to insert the electrodes. A platinum foil with a diameter of $50 \mathrm{~mm}$ and a thickness of $50 \mu \mathrm{m}$ was then assembled centrally with the disc using Neoprene glue. Holes with a $0.7 \mathrm{~mm}$ diameter were drilled through the platinum foil as a continuation of the holes in the disc. The electrodes were manufactured from a platinum wire, with a diameter of $0.5 \mathrm{~mm}$, which was coated electrophoretically using deposit of a polymeric paint. After soldering the connection cables, the electrodes were glued with an epoxy resin onto the disc such that the tops of the platinum wires just projected above the platinum foil. The wires were then rubbed down flush with the surface of the platinum foil using progressively finer grades of emery paper. The last emery paper had a grit size of $10 \mu \mathrm{m}$. The whole surface was then polished using a fine dental paste. The resulting surface roughness was about $0.11 \mu \mathrm{m}$, which is much less than the Nernst diffusion layer thickness estimated at $10 \mu \mathrm{m}$. Nickel sheets with an area of $0.15 \mathrm{~m}^{2}$ and the stainless steel tube were used as the auxiliary electrode (anode). The area of the nickel sheets and the stainless steel tube was 80 times superior to the platinum disc area. To measure only the phenomena which happen on the measuring electrode (cathode), the area of the auxiliary electrode (anode) should be large enough. Measured currents in our experiments did not change if the anode area was slightly varied which was the proof of the sufficiency of anode area.

(a)

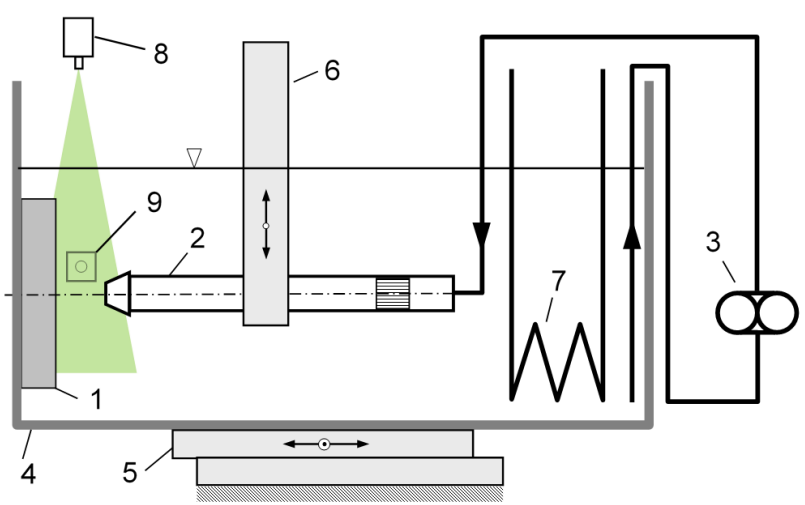

(b)

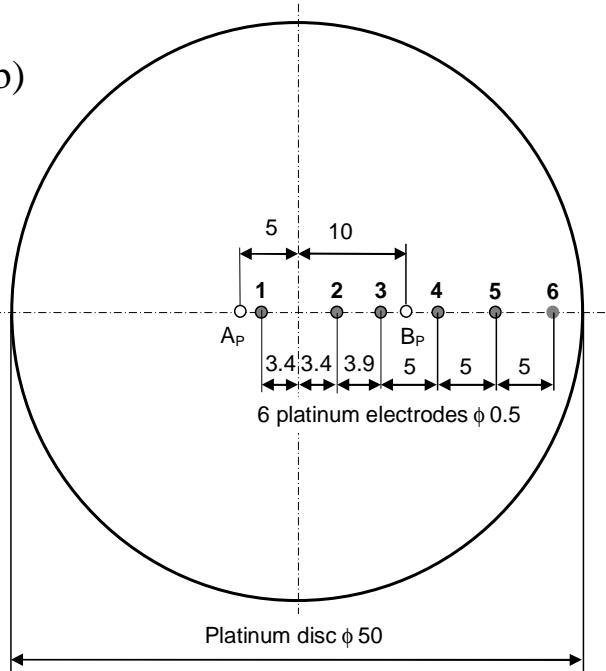

Fig. 1 a): Diagram of apparatus: 1 target disc with electrodes, 2 tube with nozzle and honeycomb, 3 pump, 4 reservoir, 5 compound table, 6 nozzle holder, 7 cooling coil, 8 laser source with laser sheet in the mild plane of the nozzle, 9 FlowSense EO camera b): Target disc with electrodes row: 1-6 electrodes $\phi 0.5 \mathrm{~mm}$. $\mathrm{A}_{\mathrm{P}}$ and $\mathrm{B}_{\mathrm{P}}$ are the limits of stagnation point displacement. 
The test fluid was an aqueous solution of $5 \mathrm{~mol} / \mathrm{m}^{3}$ potassium ferricyanide $\left(\mathrm{K}_{3}\left(\mathrm{Fe}(\mathrm{CN})_{6}\right)\right), 25 \mathrm{~mol} / \mathrm{m}^{3}$ potassium ferrocyanide $\left(\mathrm{K}_{4}\left(\mathrm{Fe}(\mathrm{CN})_{6}\right)\right)$ and $1.5 \%$ mass potassium sulphate $\left(\mathrm{K}_{2} \mathrm{SO}_{4}\right)$ as supporting electrolyte. The solution had a density of $1006 \mathrm{~kg} / \mathrm{m}^{3}$, kinematic viscosity $1.06 \cdot 10^{-6} \mathrm{~m}^{2} / \mathrm{s}$ and diffusivity $7.5 \cdot 10^{-10} \mathrm{~m}^{2} / \mathrm{s}$ at $20^{\circ} \mathrm{C}$. The resulting Schmidt number $(S c)$ was $1.420 \times 10^{3}$. The number $(n)$ of electrons involved in the electrochemical reaction was 1 .

In this study, two Cross-Shaped orifice jets were compared to a reference convergent jet (designated by CONV). The convergent nozzle had an exit diameter of $7.8 \mathrm{~mm}$ with a $4: 1$ area contraction on a length of $17 \mathrm{~mm}$. Its sketch and others details of the experimental setup can be found in Kristiawan et al. [44] and Meslem et al. [48]. Schematics of the two Cross-Shaped nozzles are shown in Figure 2. They are given by the projection of a plane Cross-Shaped orifice Fig. $2 b$ onto a plane surface (Cross-Shaped Orifice made on a flat Plate, CO/P, see Fig. 2a), and onto a spherical surface (Cross-Shaped Orifice made on a Hemisphere, CO/H, see Fig. 2c and d). The two nozzles had the same equivalent diameter $D_{e}$ of $7.8 \mathrm{~mm}$. The Reynolds number based on this equivalent diameter and the jet bulk-velocity $W_{b}$ was $R e=5620$. The distance $H$ between the jet exit and the target wall was the same for all measurements, i.e., $H=2 D_{e}$. The coordinate system $(r, Y, Z)$ attached to the nozzle is shown in Fig. 3. As sketched in this figure, the flow field may be divided into several regions. In the neighbourhood of the stagnation point $S$, the flow spreads in radial directions parallel to the wall. The development of the flow field near the wall is typically divided in two regions: the stagnation region $\left(r / D_{\mathrm{e}}<1\right)$ associated with the turning of the mean flow, where the thickness of the boundary layer is relatively independent from the radial position, and the radial wall jet region $\left(r / D_{e}>1\right)$.

\subsection{PIV measurements}

We investigated the flow field by 2D2C PIV (two in-plane velocity components in a plane field). The experiments were carried out with a Quantel BigSky $200 \mathrm{~mJ}$ double-pulsed Nd:Yag laser and a FlowSense EO (CCD) camera with a resolution of $2048 \times 2048$ pixels and a pixel size of $7.4 \times 7.4$ $\mu \mathrm{m}^{2}$. The light sheet optics produced a laser sheet of less than $1 \mathrm{~mm}$ in thickness. The maximum acquisition frequency of the PIV system is $15 \mathrm{~Hz}$. The total field of view is about $2 D_{e} \times 6 D_{e}$ with a spatial resolution of $27 \mu \mathrm{m}$ per pixel. The seeding particles are glass hollow spheres of 9 to $13 \mu \mathrm{m}$ in diameter and $1.1 \mathrm{~g} / \mathrm{cm}^{3}$ in density. 


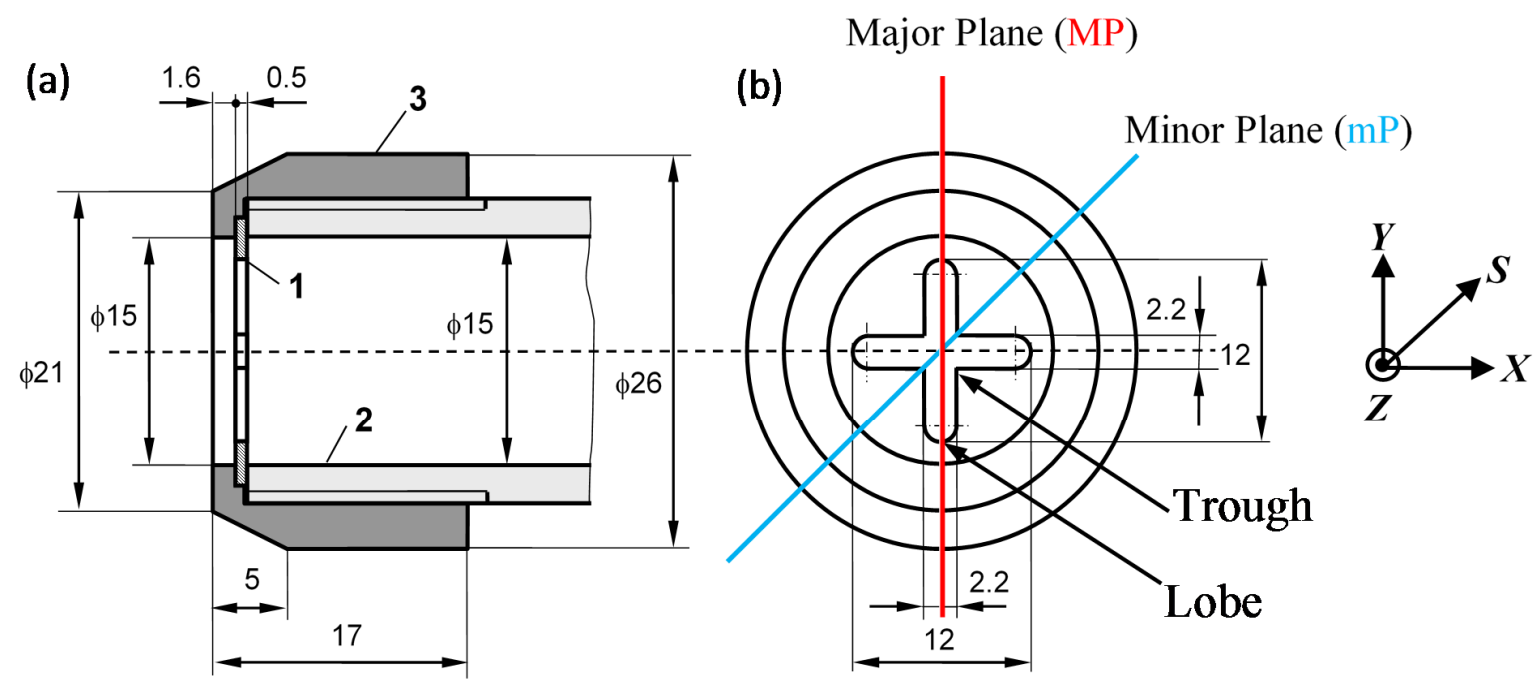

(c)

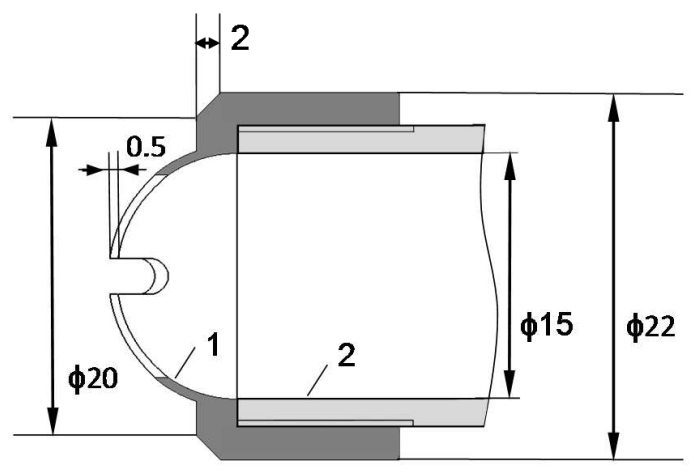

(d)

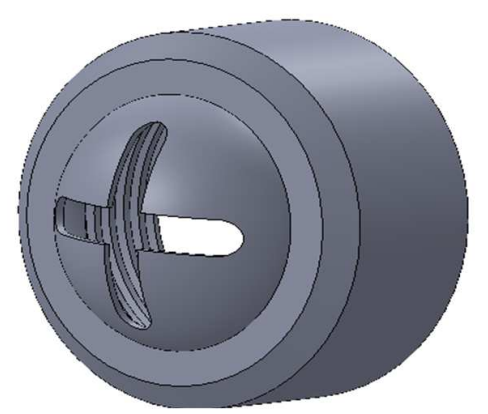

Fig. 2 Sketch of nozzles: (a) 1 - longitudinal sectional view of the cross orifice on a plane (CO/P), 2 tube; (b) - front view of CO/P; (c) 1 - longitudinal sectional view of the cross orifice on a hemisphere $(\mathrm{CO} / \mathrm{H}), 2-$ tube; $(\mathrm{d}) 3 \mathrm{D}$ view of $\mathrm{CO} / \mathrm{H}$

For each experiment, 500 couples of images were acquired. The recordings were analyzed through two different windows using Dynamics Studio Dantec software. Firstly, velocity distribution in the total field of view $\left(2 D_{e} \times 6 D_{e}\right)$ was calculated using an adaptive multi-grid correlation algorithm [49], handling the window distortion and the sub-pixel window displacement $(128 \times 128,64 \times 64$, and $32 \times 32$ pixels) and an overlap factor of $50 \%$. Secondly, to get a better spatial resolution of the velocity vectors in the radial wall jet region (Fig. 3), the same algorithm was used with a final grid composed of $8 \times 64$ pixels interrogation windows and an overlap factor of $50 \%$. The predictioncorrection validation method of the multi-grid algorithm identified, on average, less than $1 \%$ 
erroneous velocity vectors, which were replaced using a bilinear interpolation scheme. For all the experiments, the uncertainty of the measurement due to the displacement error was estimated using the theoretical analysis of Westerweel [50]. When adding the global bias errors, the total uncertainty is estimated to be in the range of 2 to $3.5 \%$ outside the boundary layer. Uncertainty rises near the impinging plate due to laser scattering reflexion, so that the boundary layer is not accessible using PIV technique. This difficulty is bypassed using another measurement technique, electrodiffusion, which is described in the next section.

PIV measurements were performed in the longitudinal plane of each jet (Fig. 1). For the crossshaped orifice jets, the measurements were carried out in two longitudinal planes, i.e., the major plane which intercept two nozzle lobes and the minor plane which intercept two nozzle troughs (Fig. 2). First, the laser sheet is adjusted into the minor plane mP. The symmetry of the measured velocity profile is checked. This assure us that the position of the first measurement plane relative to the laser sheet is correct. Then, the position of the $\mathrm{mP}$ is reported on the nozzle holder (see Fig. 1) by drawing two marks on it. These marks allow the adjustment of the major plane MP of the nozzle on the laser sheet, obtained by $45^{\circ}$ rotation of the tube supporting the nozzle relative to its initial position. Finally, the symmetry of the velocity profile in the MP is an indicator of the accuracy of the procedure. A supplementary indicator is the well-matched mean axial velocity curves, extracted from the two measurement planes MP and mP, respectively.

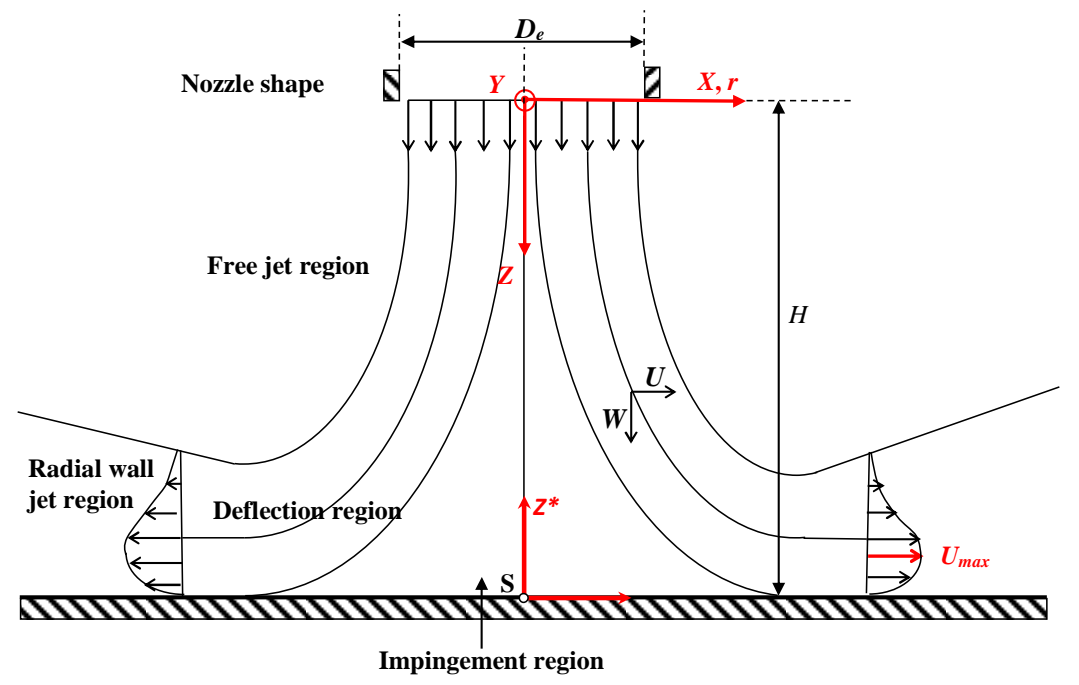

Fig. 3: Schematic description of the impinging jet on a flat plate and associated system coordinates 


\subsection{Electrodiffusion measurements}

\section{Wall shear rate}

The electrodiffusion (ED) method for wall shear rates and/or stress measurement is based on the measurement of the limiting diffusion current passing through a working electrode flush mounted on a wall. This technique has been extensively described in Kristiawan et al. [44], El Hassan et al. [41], and Meslem et al. [48], so only a brief summary will be given here. The technique was developed to measure the average rate of mass transfer and their fluctuations on a wall. It is based on the electrochemical redox reaction whose rate is limited by mass transfer on a working electrode. For the total current passing through a circular electrode in a viscosimetric flow with a uniform wall-shear rate $\gamma$, the formula corresponding to Leveque's equivalent equation for heat transfer was established by Reiss et al. [51] and is given by:

$I_{L}=\frac{0.884 \pi}{3^{1 / 3} \Gamma(4 / 3)} n F C \gamma^{1 / 3} D_{C}^{2 / 3} R^{5 / 3}$

where $C$ is the bulk concentration, $D_{C}$ the diffusion coefficient for active species, $F$ the Faraday constant, $n$ the number of electrons involved in the electrochemical reaction, $R$ the radius of the electrode and $\Gamma$ the gamma function.

It should be remembered that the basic assumption used to derive the Leveque relationship (Eq.1) is that the probe is in a flow with parallel streamlines and a uniform wall-shear rate $\gamma$. In the case of an impinging jet, the streamlines in the vicinity of a wall spread radially from the stagnation point and the wall-shear rate increases with $r$. Kristiawan et al. [44] determined the drawbacks of applying this equation to the stagnation region for an electrode with $R=0.25 \mathrm{~mm}$. At a radial distance $r=1 \mathrm{~mm}$ from the stagnation point, the authors found an error of $2.1 \%$ in wall shear rate. Taking into account the other parameters which can affect it, Meslem et al. [48] concluded that the error in wall shear rate using Eq.1 was less than $5 \%$ for $r / R \geq 4$.

\section{Mass transfer}

The limiting diffusion current is controlled by the transfer of active species to the working electrode. Under the assumption that the transfer resistance on the auxiliary electrode is negligible in comparison with that on the working electrode, the coefficient of mass transfer can be calculated using the following relation: 


$$
k=\frac{I}{S_{e l} F C}
$$

where $S_{e l}$ is the active surface of the working electrode.

The Sherwood number is then defined by:

$$
S h=\frac{k D_{e}}{D_{C}}
$$

\section{Results and discussion}

\subsection{Initial conditions}

In order to characterize the imprint of nozzle geometry on jet outlet conditions, the radial distributions of mean streamwise velocity together with streamwise and spanwise turbulence intensity based on PIV measurements are given in Fig. 4 for $Z=0.25 D_{e}$. Due to the laser light scattering on the nozzle surface, velocity measurements using PIV upstream the axial position $Z=$ $0.25 D_{e}$ could be subjected to large uncertainties. That is why the inlet velocity $\mathrm{W}_{0}$ was defined at this position (see Table 1).

Owing to the flow symmetry in each plane, only half-profiles were plotted. The shapes of these profiles depend on the geometry of the nozzle exit. The mean streamwise profile of the convergent nozzle is flat (Fig.4 a) while for the Cross-Shaped nozzles, a two-step distribution characterized the major plane (Fig. 4 a). The same behaviour was also observed by Nastase et al. [52] in a free jet from a cross-shaped orifice and by $\mathrm{Hu}$ et al. [53] in a six-lobed nozzle jet. This is because in the lobed jets, the major plane has two shear layers: the inner shear layer between the jet central core and the lobes, and the outer shear layer between the jet flow and its ambience. The junction region between the central core flow and the lobe flow appears as a velocity deficit zone. In the minor plane $(\mathrm{mP})$, the mean velocity profile was similar to that of a convergent nozzle but with a smaller diameter (Fig.4 a). The corresponding streamwise turbulence intensity profiles were also similar (Fig.4 b) with a very low value (2\%) at the jet center and a sharp peak toward the shear layer. In the major plane (MP), due to the presence of two shear layers (inner and outer), there were two peaks in the corresponding turbulence profiles. A wide region of intensive mixing characterized by 
high turbulence intensity (around 30\% of $W_{0}$ ) could be observed with jets from the $\mathrm{CO} / \mathrm{P}$ and $\mathrm{CO} / \mathrm{H}$ nozzles. Nearly identical turbulence profiles were measured by Hu et al. [53] in their six-lobed jet. Spanwise turbulence intensity distribution for the convergent nozzle was uniformly low across the jet, as expected (Fig.4 c). Similarly, low values were also obtained with the CO/P nozzle jet, except in the shear layer of the minor plane. In the $\mathrm{CO} / \mathrm{H}$ nozzle jet, the curvature of the orifice led to a relatively high spanwise turbulence intensity in the major plane whereas in the minor plane, it was as low as in the reference convergent jet. The particular distribution of spanwise turbulence intensity at the exit of each lobed jet was undoubtedly linked to the development of their respective streamwise vortices. As was emphasized by Nastase et al. [54], the major difference between the plate and the hemispherical lobed orifice flows lies in the different positions of the secondary streamwise structures. In the jet issued from a plane lobed orifice, streamwise vortices develop in the lobe troughs and this leads to axis-switching phenomena, i.e. a $45^{\circ}$-like rotation of the initial transverse lobed field. The rotation angle is $45^{\circ}$ if the lobed jet is cross-shaped $[55,56]$. In a hemispherical lobed orifice, the curvature of the surface completely changes the behaviour of the jet flow that is generated. No axis-switching occurs, i.e. the jet conserves the shape imposed initially by the nozzle, with the major and the minor axes oriented in the same direction as those of the nozzle, with streamwise secondary vortices localized on the top of each lobe [54].

Table 1: Initial experimental conditions of the three flows studied

\begin{tabular}{|c|c|c|c|c|c|c|c|}
\hline $\mathbf{N}$ & \multicolumn{2}{|c|}{ Symbol } & $\begin{array}{c}\mathrm{W}_{0}= \\
\mathrm{W}\left(\mathrm{Z}=0.25 \mathrm{D}_{\mathrm{e}}\right) \\
(\mathrm{m} / \mathrm{s})\end{array}$ & $\mathrm{W}_{0} / \mathrm{W}_{\mathrm{b}}$ & $\delta_{0}^{*} / \mathrm{D}_{\mathrm{e}}$ & $\theta_{0} / \mathrm{D}_{\mathrm{e}}$ & $\begin{array}{l}\mathrm{H}^{*}= \\
\delta_{0}^{*} / \theta_{0}\end{array}$ \\
\hline CONV & & & 0.817 & 1.06 & 0.059 & 0.022 & 2.68 \\
\hline \multirow{2}{*}{$\mathrm{CO} / \mathrm{P}$} & (MP) & & 1.20 & 1.56 & 0.068 & 0.025 & 2.73 \\
\hline & $(\mathbf{m P})$ & -8 & 1.20 & 1.56 & 0.061 & 0.022 & 2.77 \\
\hline \multirow{2}{*}{$\mathrm{CO} / \mathrm{H}$} & (MP) & $-e_{-1}$ & 1.45 & 1.89 & 0.153 & 0.055 & 2.79 \\
\hline & $(\mathbf{m P})$ & & 1.45 & 1.89 & 0.043 & 0.016 & 2.69 \\
\hline
\end{tabular}

N: Nozzle; CONV: Convergent; CO/P: Cross-shaped Orifice on a plane; CO/H: Cross-shaped Orifice on a hemisphere 
(a)
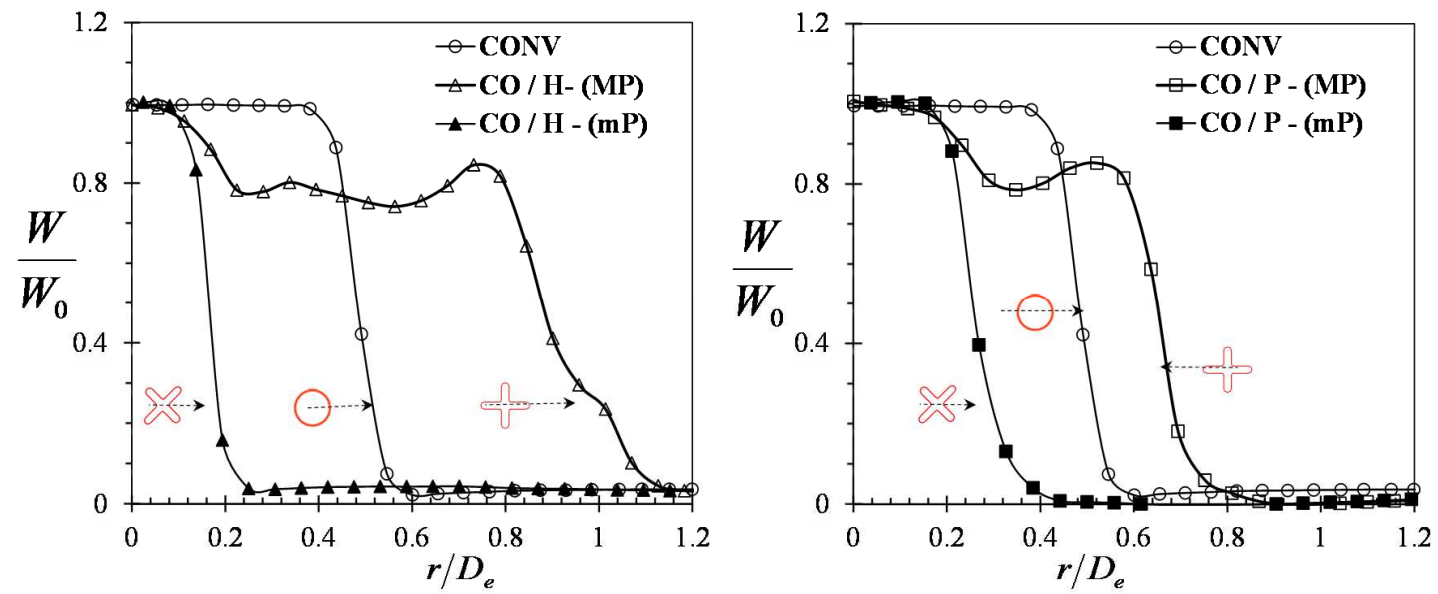

(b)
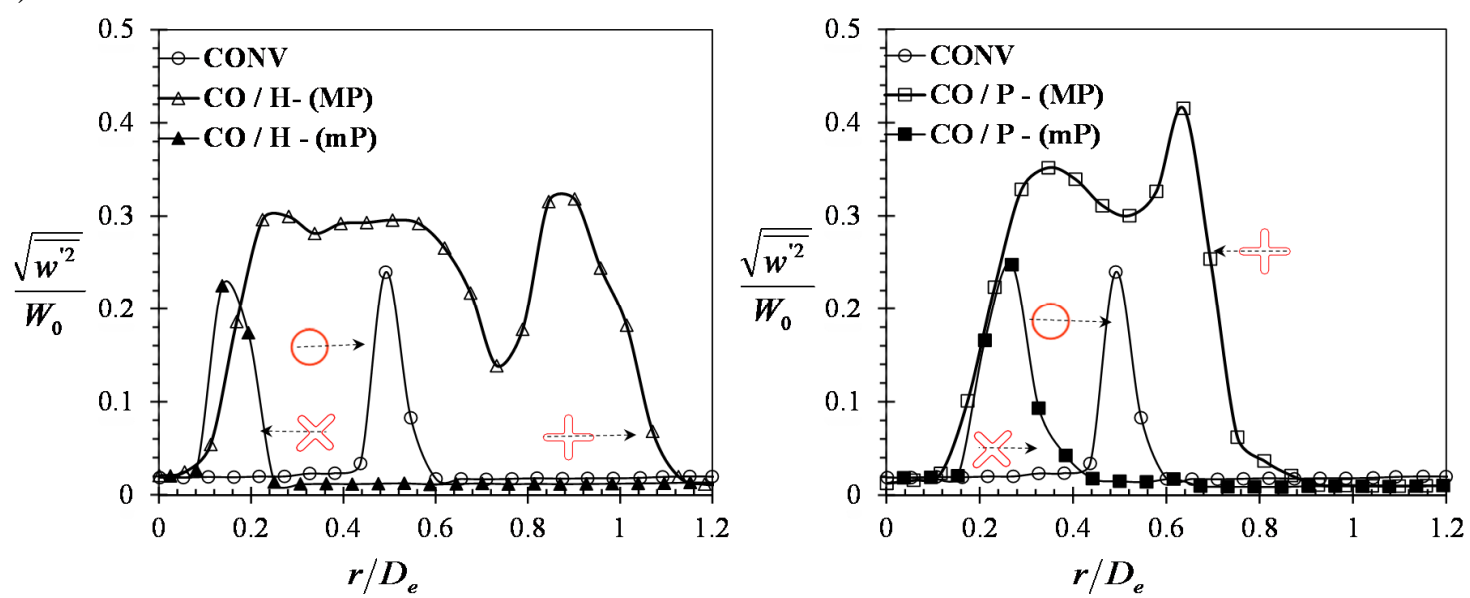

(c)
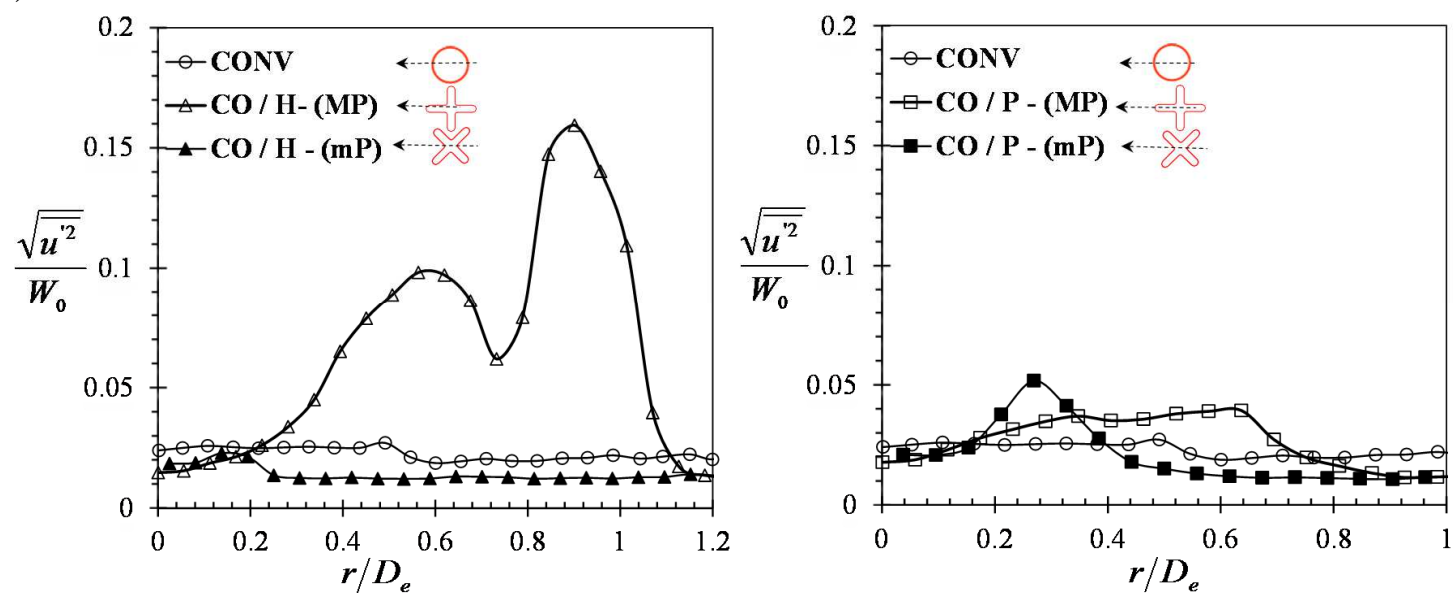

Fig.4 Radial distribution at the jet exit $Z=0.25 D_{e}$ of the mean axial velocity $W$ at $\operatorname{Re}_{\mathrm{b}}=5620$ (a), the rms axial velocity $\sqrt{\overline{w^{\prime 2}}} / W_{0}(\mathrm{~b})$, and the rms radial velocity $\sqrt{\overline{u^{\prime 2}}} / W_{0}$ (c) 
Details on the initial momentum thickness and outlet velocity are summarized in Table 1 . The momentum thickness for the jet flowing from the CONV and CO/P nozzles in non-dimensional form $\theta_{0} / D_{\mathrm{e}}$ ranged from 0.022 to 0.026 . These values fall within the range reported in the literature [29]. A typical value was obtained for $\theta_{0} / D_{e}$ in the minor plane for the jet from the $\mathrm{CO} / \mathrm{H}$ nozzle, whereas a high value $(0.055)$, which was outside the usual range, was observed in the major plane due to flow stretching. Flow acceleration at the jet exit is another initial parameter which has a major influence on the mechanisms of local heat/mass transfer at the target wall. The ratio of maximum centreline velocity $W_{0}$ and jet bulk velocity $W_{b}$ highlights the level of flow acceleration as a function of the nozzle geometry. The flow with greatest acceleration was achieved with the $\mathrm{CO} / \mathrm{H}$ nozzle, followed by the $\mathrm{CO} / \mathrm{P}$ nozzle and then the CONV nozzle.

\subsection{Flow characteristics}

In this section the general features of the two Cross-Shaped orifice impinging jets are examined and compared to the reference convergent jet. The analysis focuses on both the free and wall jet regions defined in Fig. 3. For wall jet region analysis, the axis $Z *$ taking its origin on the target wall $\left(Z^{*}=H-Z\right)$ has been introduced.

In Figs 5 and 6, the spatial developments of the two Cross-Shaped orifice jets in their respective major and minor planes are compared to the reference convergent jet in its longitudinal plane. To highlight the recirculation regions, if there were any, streamlines (in blue) are superimposed on the mean vector field in Fig. 5. Atypical behaviour was seen in the minor plane $(\mathrm{mP})$ of the $\mathrm{CO} / \mathrm{H}$ nozzle jet: a large recirculation zone was visible in the deflection region on either side of the jetaxis. In addition, the wall jet in the $\mathrm{mP}$ was spread much more widely than in the major plane (MP) of the same jet. Fig. 6 gives the profiles of the velocity vector in the free jet region and in the radial wall jet region, respectively. In order to achieve a better visualization of the wall flow region and to confirm the previous observation, these profiles were superimposed on the contour (colormap) of the radial velocity component. The thickness of the wall jet flow in the $\mathrm{mP}$ of $\mathrm{CO} / \mathrm{H}$ was nearly five times the thickness in the other planes (i.e. the $\mathrm{MP}$ in $\mathrm{CO} / \mathrm{H}$, the $\mathrm{mP}$ and $\mathrm{MP}$ in $\mathrm{CO} / \mathrm{P}$ and in $\mathrm{CONV}$ ). Another feature of the jet from the $\mathrm{CO} / \mathrm{H}$ nozzle was the conservation of the double shear 
layer in its MP as far as the stagnation region. This happened because a high radial outward velocity component was present in the outer shear layer of the free jet region. The curvature of the lobes generated outward movement and hence a large expansion of the jet in the MP. In this plane, jet spreading in the stagnation region was almost twice as large as at the jet exit. Large spreading was also seen in the CO/P nozzle jet, although not in the MP, as was the case for the CO/H nozzle, but in the $\mathrm{mP}$ due to the axis-switching phenomenon. Such a phenomena has been observed in our previous studies of free planar lobed orifice jets $[52,55]$ and is reported in the recent numerical study on a low aspect ratio rectangular impinging jet of Gorman et al. [57]. It should be noted that the axis-switching phenomenon is not more reported in the literature in the case of the impinging jets. During the deformation process, the inner shear layer of the MP disappeared progressively as it moved downstream along the jet axis. It should be noted that the axis-switching process in the present study could not be completed due to the presence of the target wall at $H=2 D_{e}$ from the nozzle. Indeed, it was observed that the axis-switching process ended at $3 D_{e}$ from the exit in the free air-air Cross-Shaped jet from same orifices $[52,55]$. Around $2 D_{e}$ from the nozzle exit, the free $\mathrm{CO} / \mathrm{P}$ nozzle jet [55] and the impinging CO/P nozzle jet (present study, see Fig. 6b), had the same thickness in the minor and major planes. A comparison of the jet fields in the cases of the $\mathrm{CO} / \mathrm{P}$ and $\mathrm{CO} / \mathrm{H}$ nozzles (Fig. $6 \mathrm{~b}$ and c) led us to affirm that introducing a curvature of the surface containing a lobed orifice completely changes the behaviour of the jet flow generated. Specifically, the curvature of the lobes in the $\mathrm{CO} / \mathrm{H}$ nozzle conserves the asymmetry of the flow. 


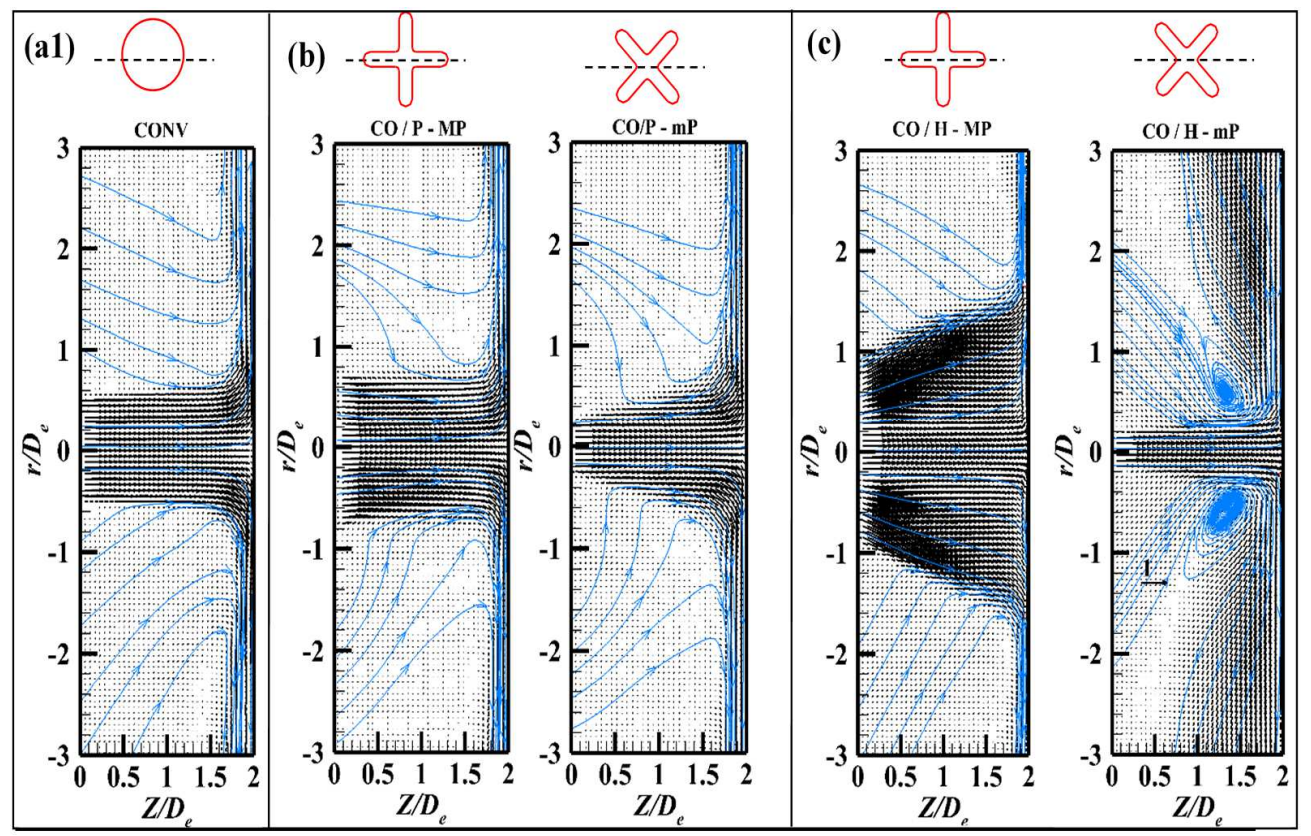

Fig.5 Mean vector fields $\left(\frac{W}{W_{o}} \overrightarrow{e_{z}}+\frac{U_{r}}{W_{o}} \overrightarrow{e_{r}}\right)$ and the corresponding streamlines (in blue): convergent nozzle (a), cross-shaped orifice on a plane (b), cross-shaped orifice on a hemisphere (c)

As already observed by Nastase et al. [54] when studying a free jet from a six lobed (daisy) hemispherical nozzle, the axis switching phenomenon does not occur in a hemispherical lobed nozzle jet. They showed that the daisy hemispherical nozzle jet entrains almost six times as much surrounding fluid as its counterpart daisy planar orifice nozzle jet, and this entrainment occurs mainly in nozzle trough regions (i.e. in the minor planes of the nozzle). In the context of the present study, the target wall confined the strong self-induction of the lobed hemispherical nozzle jet. As a result, permanent large recirculation vortices were formed in the $\mathrm{mP}$ in the region delimited by $Z / D_{e}= \pm 1.4$ and $r / D_{e}= \pm 0.6$. Theses vortices generated a strong fluid ejection which fed the $\mathrm{mP}$ radial wall region of the jet. This explains in part, the thickness observed in the minor plane of the radial wall region with the jet from the $\mathrm{CO} / \mathrm{H}$ nozzle (Fig. $6 \mathrm{c}$ ).

Flow spreading and mean velocity decay in the free region of jets from the $\mathrm{CO} / \mathrm{P}$ and $\mathrm{CO} / \mathrm{H}$ nozzles are shown in Figs 7 and 8 a. The data for the reference convergent nozzle jet (CONV) are shown for comparison. The jet widths in the major plane $X_{0.1}$ and in the minor plane $S_{0.1}$ were defined as 
the distance from the centreline to the point where the mean axial velocity reached $10 \%$ of its centreline value.

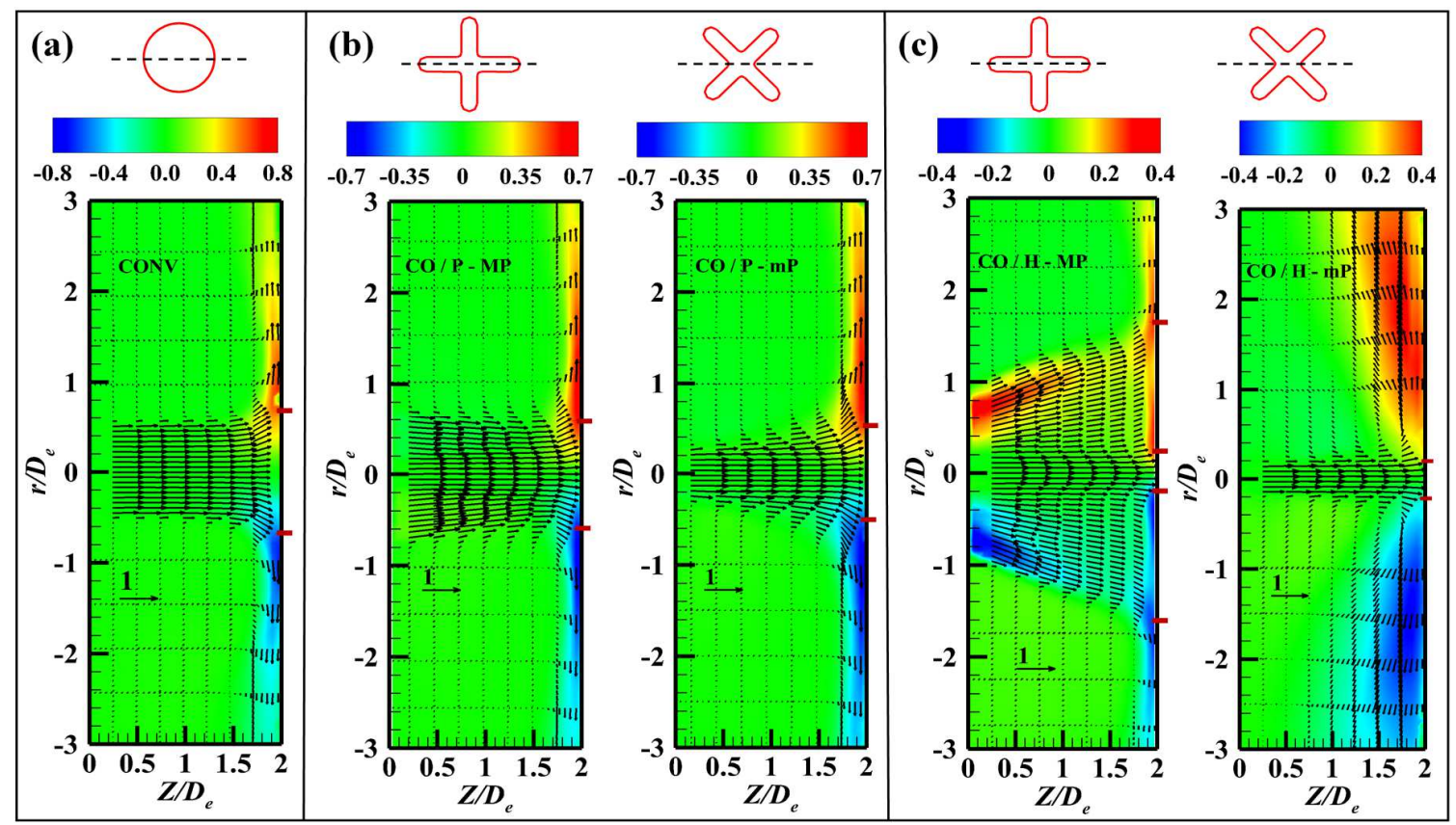

Fig.6 Vector profiles $\left(\frac{W}{W_{0}} \overrightarrow{e_{Z}}+\frac{U}{W_{0}} \overrightarrow{e_{r}}\right)$ at different axial and radial positions $\left(Z / D_{e}\right.$ and $\left.r / D_{e}\right)$, and radial velocity $\frac{U}{W_{0}}$ contours (colormap): convergent nozzle (a), cross-shaped orifice on a plane (b), cross-shaped orifice on a hemisphere (c). The red ticks indicate the positions of maximum wall shear rate $\gamma_{\max }$

The tendency towards axis-switching in the $\mathrm{CO} / \mathrm{P}$ nozzle jet and the absence of axis-switching in the $\mathrm{CO} / \mathrm{H}$ nozzle jet are well described by the changes in their respective jet widths $X_{0.1}$ and $S_{0.1}$ (Fig. $7 \mathrm{a}$ and b). In the case of $\mathrm{CO} / \mathrm{P}, X_{0.1}$ was approximately constant in the MP, whereas in the $\mathrm{mP} S_{0.1}$ increased rapidly. The resulting cross over was aborted by flow deflection due to the impinging plate. For $\mathrm{CO} / \mathrm{H}$, jet width increased in both the $\mathrm{mP}$ and the MP. However, $S_{0.1}$ in the $\mathrm{mP}$ decreased somewhat near the impinging plate due to flow contraction by large recirculation zones evidenced in Fig. 5 c. 

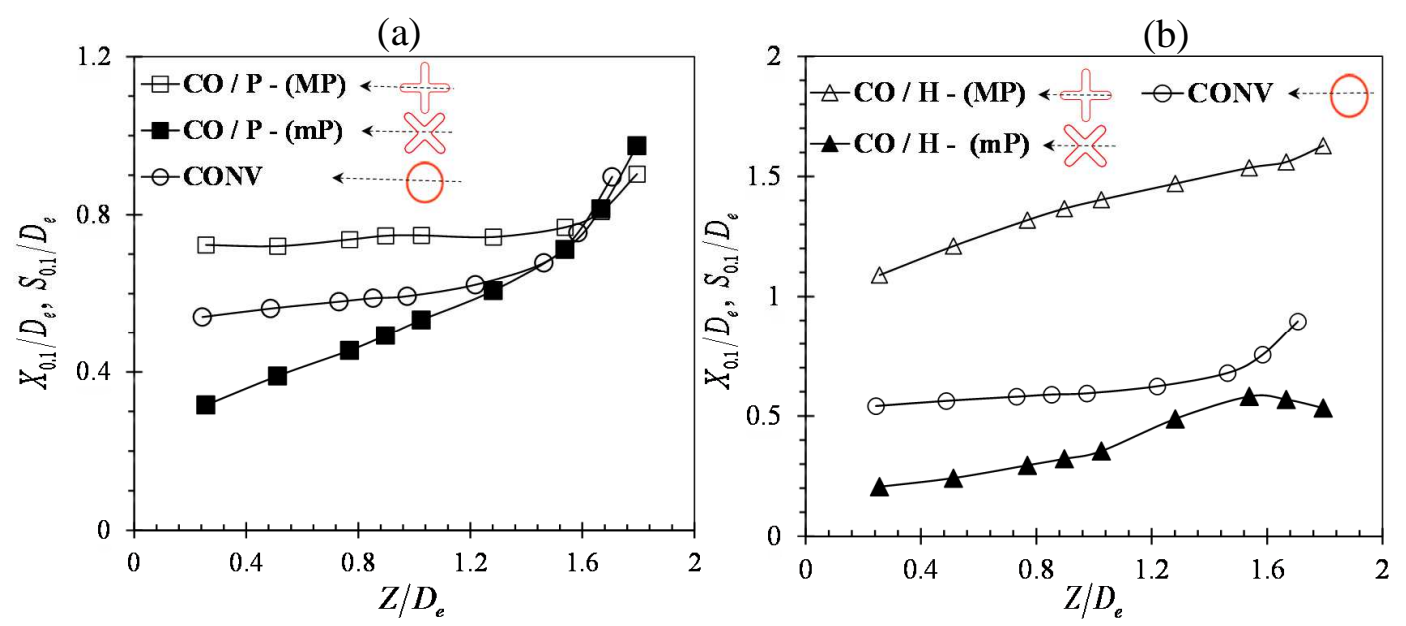

Fig.7 Growth of the outer edge of the shear layer: (a) Comparison between convergent nozzle and Cross-Shaped orifice on a plane; (b) Comparison between convergent nozzle and Cross-Shaped orifice on a hemisphere

Normalized axial mean velocity and turbulence intensity distributions along the jet centerline are given in Fig. 8 a and b. The subfigures compare our round convergent jet data to those of Baydar and Ozmen [58] obtained with a round pipe impinging jet at a high Reynolds number of $3 \times 10^{4}$. For the mean axial velocity, the close agreement between the two curves is not surprising. Indeed, though there may be differences between the characteristics of the two round jets due to differences in the shape of the corresponding nozzles [1] or due to a different Reynolds number [59], the differences are imperceptible on the axis in the region of the first two diameters from the nozzle exit. Defining the jet potential core length as the streamwise distance from the jet exit where the axial velocity decreases to $95 \%$ of its initial value, a length of $1.24 D_{e}$ was found for our impinging convergent jet, which is equal to that of the impinging pipe jet of Baydar and Ozmen [58].

As shown in Fig. 8 a, a drop in velocity in the $\mathrm{CO} / \mathrm{H}$ nozzle jet was delayed compared to the $\mathrm{CO} / \mathrm{P}$ nozzle jet when approaching the wall. This behaviour could be related to the imprint of the permanent recirculation vortices in the $\mathrm{CO} / \mathrm{H}$ nozzle jet (Fig. $5 \mathrm{c}$ ) that pinch the flow and hence delaying the decrease in the axial velocity when the jet approaches the wall.

The change in the axial turbulence intensity along the jet centerline in Fig. 8 b could be linked to the mean axial velocity decay in Fig. 8 a. Each curve in the subfigure has one sharp peak in the vicinity of the wall and then decreases. Coopers et al. [60] associated the aforementioned behaviour to the damping effects exerted by the wall. The similarity between our results and those of Baydar and Ozmen on the circular jet, strongly suggests that our PIV measurements are reliable. The 
limitation of the PIV technique in the vicinity of the wall reflects the fact that the turbulence intensity didn't reach zero.

Taking into consideration all the three jets used in the present investigation, the axial turbulence intensity at their nozzle exit was low, with a value around $2 \%$ of the maximum outlet velocity $W_{0}$ (Fig. 8 b). As the impingement plate was approached the turbulence intensity increased, in contrast to mean axial velocity (Fig. 8 a). However, the Cross-Shaped orifice jets exhibited a marked difference compared with the convergent jet. In the CO/P nozzle jet, two peaks were identified on the axial fluctuation. Whereas the second peak is believed to be related to the wall damping effect [60], the occurrence of the first peak is not fully understood. From the study of Zaman et al. [13] on a free rectangular lobed jet, the appearance of a sharp peak on the axial turbulence intensity near the jet exit could be related to the merging of the shear layers shed from an individual lobe. In fact, the authors observed that the distance of the first peak from the jet exit, scales on the width of the lobes $\left(a_{1}\right)$, with $4 \leq Z / a_{1} \leq 6$. In our case, the first peak in the $\mathrm{CO} / \mathrm{P}$ nozzle jet occurred at $Z / a_{1}=5.2$, which falls within the reported range [13].

No peak was visible on the axial fluctuation in the $\mathrm{CO} / \mathrm{H}$ nozzle jet (Fig. 8 b). Turbulence increased monotonically from $Z=0.4 D_{e}$ towards the wall at a level that was twice as high, on average, as that of the $\mathrm{CO} / \mathrm{P}$ nozzle jet. The high level of jet centreline fluctuations in the $\mathrm{CO} / \mathrm{H}$ nozzle jet is probably related to flow agitation by the numerous vortices generated in this jet that penetrate deeply into the jet core (see Fig.8 c). This particular behaviour is undoubtedly related to the hemispherical geometry which introduces supplementary shear to that originating from the corrugated nozzle lip.

To characterize quantitatively the wall jet region, mean radial velocity profiles at $r=1 D_{e}$ and $r=2 D_{e}$ were plotted, as shown in Fig.9. In the main figures, the mean radial velocity $U$ was normalized with the streamwise exit velocity $W_{0}$ and plotted against $Z * / D_{e}$ (the $Z *$ axis takes its origin on the target wall, see Fig. 3). In order to make a comparison with the theoretical solution of Glauert [60], the mean radial velocity $U$ was normalized with the corresponding maximum radial velocity $U_{\max }$, and plotted against $Z * / Z *_{0.5}$, as shown in the subfigures of Fig. $9, Z^{*} *_{0.5}$ is the $Z *$ position in the wall jet where the radial velocity $U$ takes the value $0.5 U_{\max }$.

As shown in Fig. 9 a, at the radial position $r=1 D_{\mathrm{e}}$ all the profiles for Cross-Shaped nozzles were, to a lesser extent, far from the reference convergent nozzle profile. The largest deviation from the convergent nozzle profile was found in the $\mathrm{CO} / \mathrm{H}$ nozzle jet. The maximum normalized velocity 
$U / W_{0}$ at this radial position was 0.2 and 0.4 for the MP and $\mathrm{mP}$, respectively, in comparison to 0.8 for the reference nozzle jet CONV.

(c)

(a)

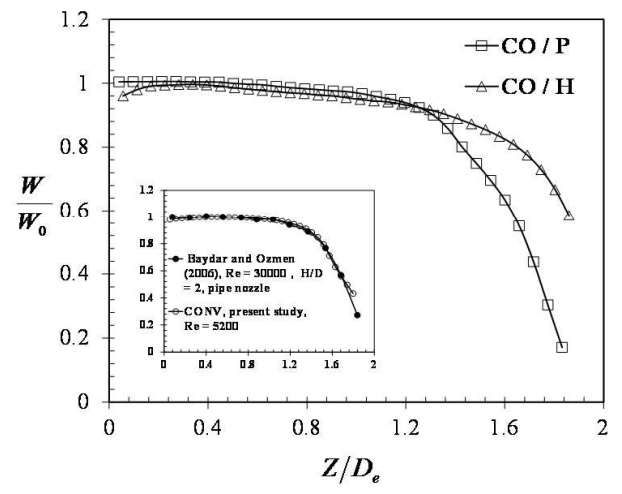

(b)

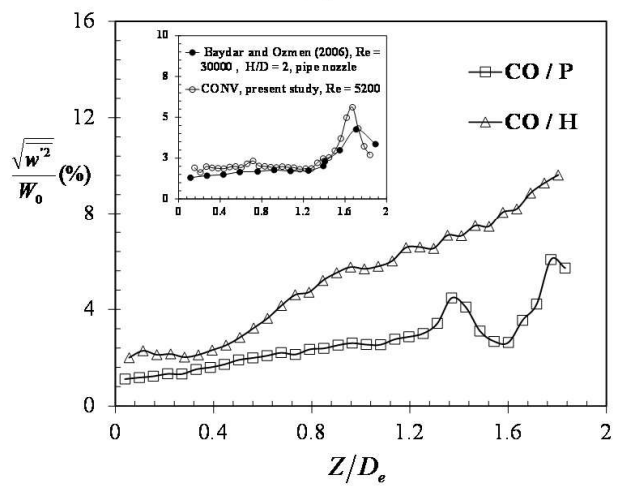

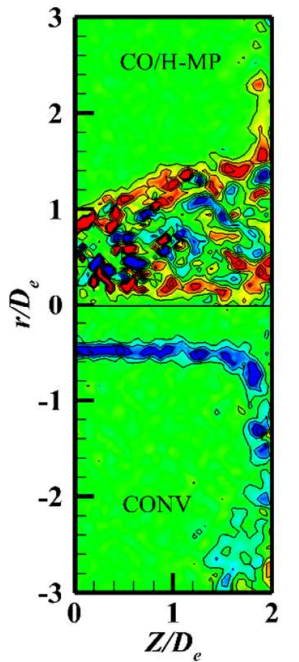

Fig.8 Normalized axial mean velocity (a) and turbulence intensity (b) distributions along the jet centerline in the three cases studied - the convergent jet (CONV) is compared to the literature [56]. (c) Contours of Instantaneous Azimuthal Vorticity $\omega_{Y}=\left(\frac{\partial U}{\partial Z}-\frac{\partial W}{\partial r}\right)$

The two-step distribution observed in the free jet MP region of the $\mathrm{CO} / \mathrm{H}$ nozzle jet (Fig. $6 \mathrm{c}$ ) was preserved and the trace of that two-step distribution was also visible in the wall jet region. In the $\mathrm{MP}$ of the $\mathrm{CO} / \mathrm{H}$ nozzle jet, the $r=1 D_{\mathrm{e}}$ position fell in the deficit zone of the wall jet region, which is the trace of the junction in the free jet region between the central core flow and the lobe flow. A greater expansion of the wall jet region was observed in the $\mathrm{mP}$ of the $\mathrm{CO} / \mathrm{H}$ nozzle jet than in the MP (Fig. 6 c and Fig. 9).

It is well known that a rectangular impinging jet on a flat plate will expand only on transversal direction [61]. Due to the fact that the cross shaped orifice is basically composed of two rectangular orifices, when there is no switching-over phenomenon in the cross-shaped jet, as it is the case in $\mathrm{CO} / \mathrm{H}$ nozzle jet, the expansion in the $\mathrm{mP}$ is a direct consequence of the flow through the two rectangular orifices composing it. In fact, the flow in each $\mathrm{MP}$ of the $\mathrm{CO} / \mathrm{H}$ nozzle jet acts as a plane impinging jet due to the absence of switching-over phenomenon and the conservation of the double shear layer until the wall target. 
In our opinion the large expansion of the boundary layer in the $\mathrm{mP}$ of $\mathrm{CO} / \mathrm{H}$ impinging nozzle jet (Fig. 6c) is due to two cumulative effects: (i) the effect described earlier of the impinging $\mathrm{CO} / \mathrm{H}$ nozzle jet through the two rectangular orifices that are composing the cross-shaped orifice, and (ii) the intense entrainment of ambient fluid in the $\mathrm{mP}$ of $\mathrm{CO} / \mathrm{H}$ jet which characterizes the hemispherical lobed jets [54]. This intense entrainment fed the corresponding boundary layer in the $\mathrm{mP}$ of the $\mathrm{CO} / \mathrm{H}$ nozzle jet.

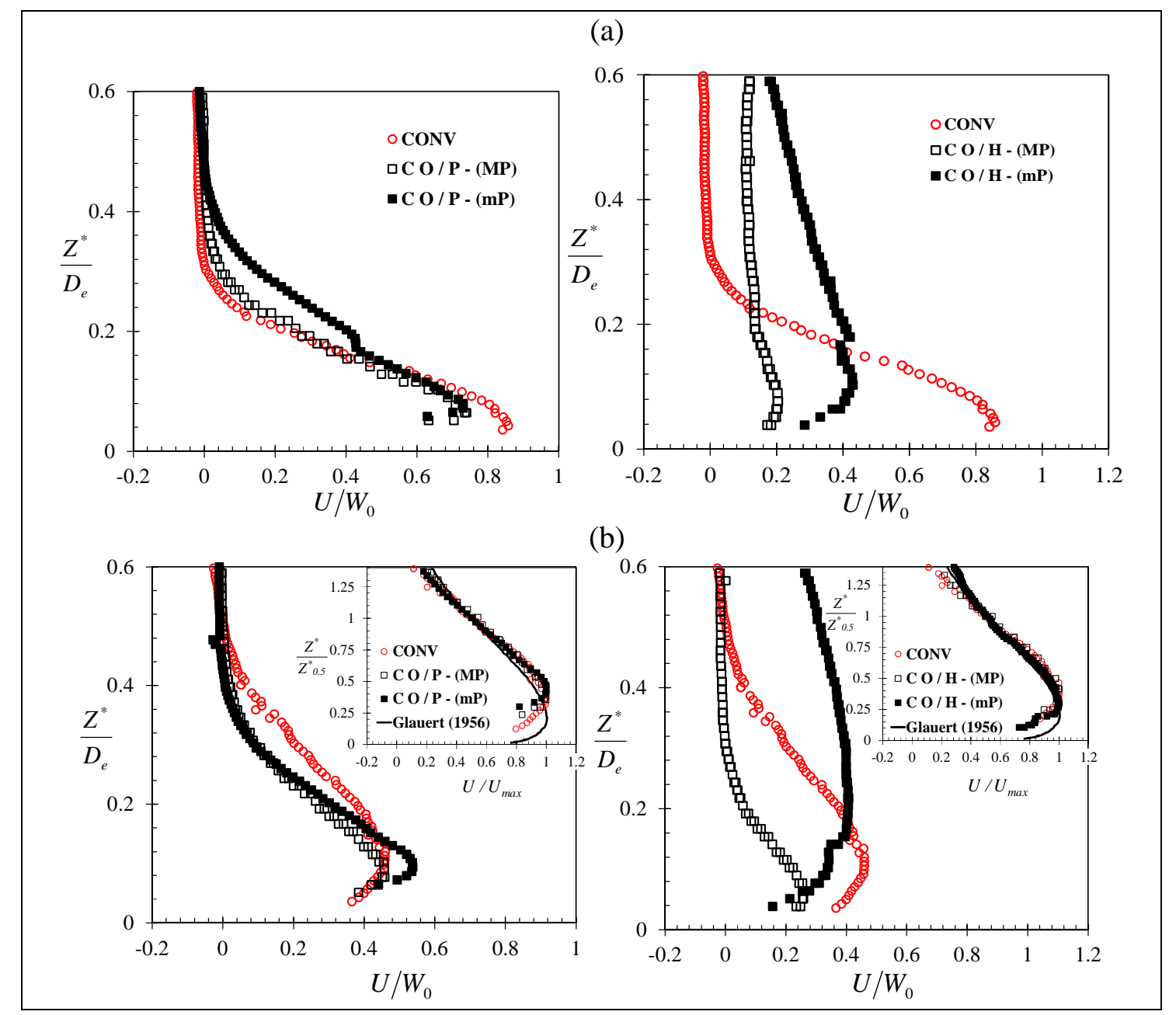

Fig.9 Normalized radial mean velocity distributions in the wall jet region at $r=1 D_{e}$ (a) and $r=2 D_{e}$ (b). Comparison to the predicted curve of Glauert [60] with $\alpha=1.3$ in the subfigures 
At the second $r=2 D_{\text {e }}$ position (see the Subfigures of Fig. $9 \mathrm{~b}$ ) all the profiles matched with the Glauert [62] solution in their outer region. The PIV measurements made in their inner region are questionable due to laser scattering by the wall surface. Nevertheless, the profiles for $\mathrm{CO} / \mathrm{P}$ were similar in the $\mathrm{mP}$ and MP planes and were close to the reference profile of the convergent nozzle (Fig.9, left). The profiles in the $\mathrm{mP}$ and $\mathrm{MP}$ for $\mathrm{CO} / \mathrm{H}$ (Fig.9, right) were different from the reference profile and also from each other.

Although the uncertainties on velocity measurements could be high near the target wall, we decided to examine the radial changes of the maximum velocity in the radial wall jet region. Fig. 10 shows the variation of the normalized maximum velocity $U_{\max } / W_{0}$ of the velocity profile in the radial wall jet region (Fig. 3) as a function of the normalized radial distance from the stagnation point. Overall, these curves confirm the previous observations from Fig. 9. The CO/P and CONV nozzles produced similar changes despite differences in magnitude (Fig. 10 a). Two regions can be identified: the first is where the radial velocity increased and it ends at the $r=1 D_{e}$ position, where the maximum radial velocity reached its highest value. In the second region, beyond $r=1 D_{e}$, maximum velocity decreased. The highest value of maximum radial velocity in the round jet was almost equal to the jet exit velocity $W_{0}$. This is consistent with the results of Tummers et al. [63] and $\mathrm{Xu}$ and Hangan [64]. In the CO/P nozzle jet, the maximum value in the region around $r=1 D_{e}$ was about $16 \%$ lower than that of the reference convergent nozzle jet. This is probably due to the fact that the lobed CO/P nozzle generates more induction than the round nozzle $[52,55]$. In the second region of the $\mathrm{CO} / \mathrm{P}$ nozzle jet, the maximum radial velocity in $\mathrm{mP}$ was higher than that in MP. This behaviour is probably due to the contribution of the streamwise structures which are developed in the troughs of the CO/P nozzle $[52,55]$.

The $\mathrm{CO} / \mathrm{H}$ nozzle jet was significantly different from the two others in terms of radial maximum velocity distribution (Fig. $10 \mathrm{~b}$ ). The magnitude of $U_{\max } / W_{0}$ was about $50 \%$ lower than in the convergent nozzle jet. The profile in the minor plane was almost flat, whereas in the major plane two small peaks were present as an imprint of the two-step velocity distribution in the free jet region (Fig. 6 c). 
(a)

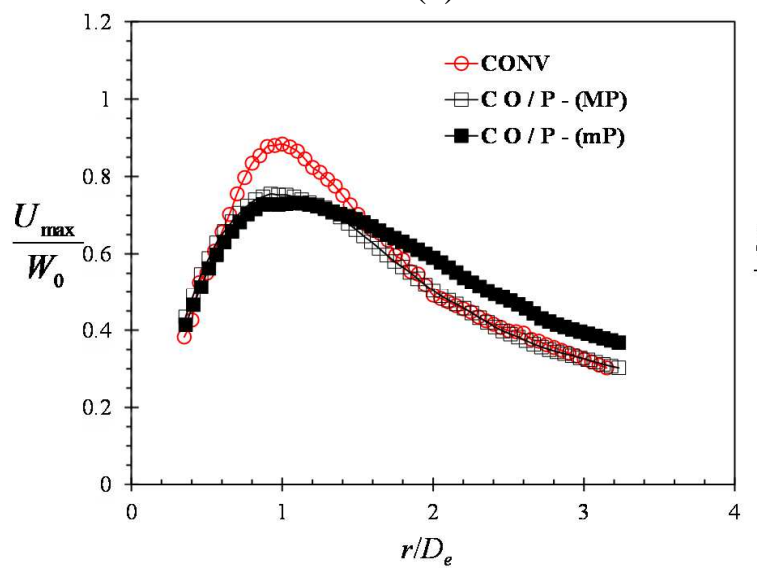

(b)

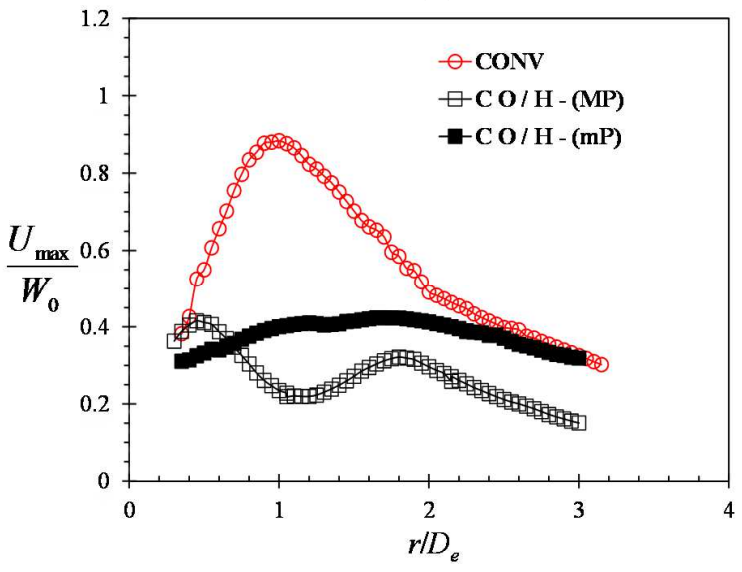

Fig.10 Normalized maximum radial mean velocity distributions: (a) $\mathrm{CO} / \mathrm{P}$ and CONV nozzles; (b) $\mathrm{CO} / \mathrm{H}$ and CONV nozzle

\subsection{Wall shear rate and mass transfer}

Local and instantaneous wall shear rate and mass transfer coefficient at the target wall were acquired separately using the electrodiffusion (ED) technique described above in paragraph 2.3. During the local wall shear rate measurements, the platinum wires worked as cathode and the platinum disc (Fig. 1 b) with the nickel sheets as anode. During the local and global mass transfer coefficient measurements the platinum wires and the platinum disc worked as cathode and the nickel sheets as anode. In this case, the platinum disc was maintained at the same potential as for the working electrodes; whereas the platinum disc was used to measure the global mass transfer coefficient, the platinum wires were used to measure the local mass transfer coefficient.

Refined radial distributions of the wall shear rate $\gamma$ and the Sherwood number $S h$ were obtained by moving the stagnation point horizontally in the range limited by the points $A_{p}$ and $B_{p}$ on the target shown in Fig. 1 b. Several values for the wall shear rate and the mass transfer coefficient measured by different electrodes at similar radial distances from the stagnation point were obtained. Given the complexity of lobed jets, thirty-one displacements of the stagnation point at $0.5 \mathrm{~mm}$ steps were performed to efficiently capture the details of the wall shear rate and Sherwood number profiles, except for the convergent nozzle jet and for the minor plane of the $\mathrm{CO} / \mathrm{H}$ nozzle jet, for which sixteen $1 \mathrm{~mm}$ step displacements were sufficient. 
The radial distribution of the mean wall shear rate of the Cross-Shaped nozzle jets are compared to that of the convergent nozzle jet in Fig.11. In both the CO/P and CONV nozzles, the wall shear rate was almost zero at the stagnation point, whereas it was relatively high in $\mathrm{CO} / \mathrm{H}$. It should be remembered that at the vicinity of the stagnation point in the $\mathrm{CO} / \mathrm{H}$ nozzle jet, the centreline streamwise turbulence intensity did not start to decrease, as occurred in the CO/P and CONV jets cases (Fig. 8 b). The strong stretching of the flow by the curved surface of the $\mathrm{CO} / \mathrm{H}$ nozzle and the resulting deep penetration of the vortices towards the jet axis (Fig.8 c) were responsible for the high turbulence (Fig. 8 b) and the high wall shear rate (Fig. $11 \mathrm{~b}$ ) in the corresponding stagnation region.

(a)

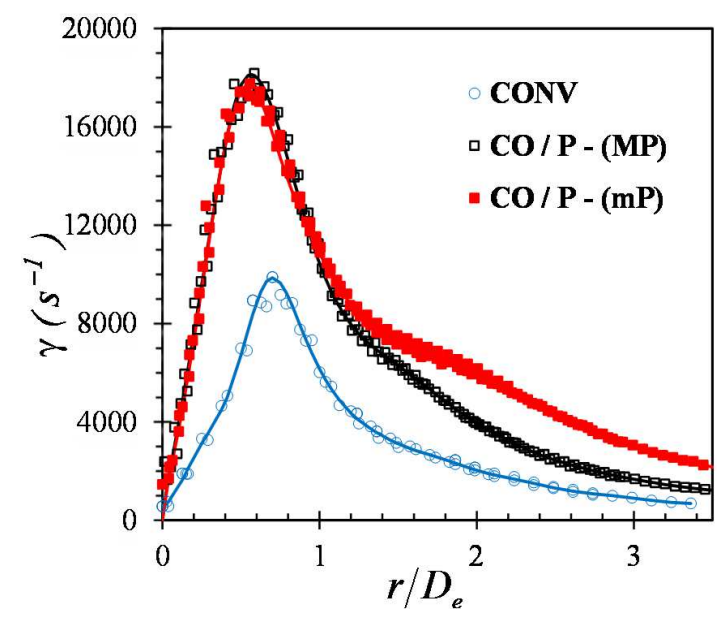

(b)

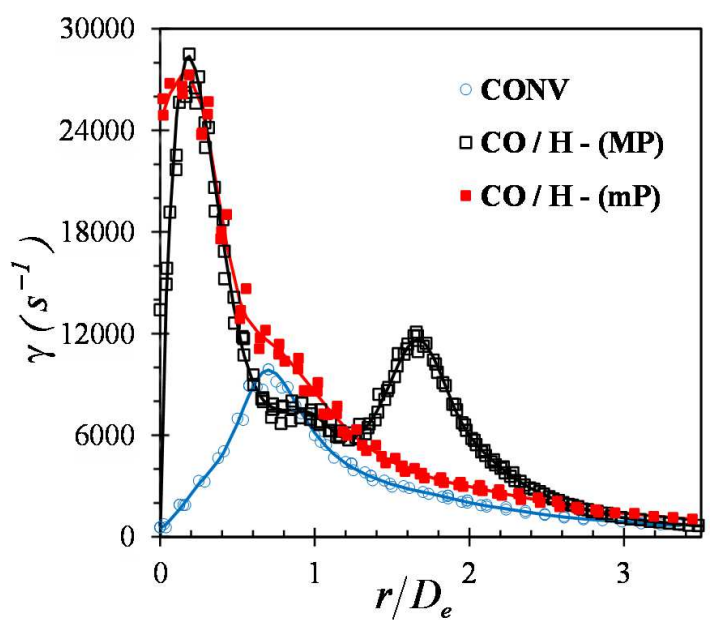

Fig.11 Mean wall-shear rate as a function of radial distance from the stagnation point: (a) CONV and $\mathrm{CO} / \mathrm{P}$ nozzles; (b) $\mathrm{CONV}$ and $\mathrm{CO} / \mathrm{H}$ nozzles - the solid lines are the best fit curves for the experimental data

In each jet, the wall shear rate increased monotonically with radial distance, reached a maximum and then decreased. In the convergent jet, the maximum $\gamma_{\max }$ occurred around $r / D_{\mathrm{e}}=0.68$, which falls in the range $0.6 D_{e}-0.74 D_{e}$ reported in the literature $[33,38,39]$ for the case of a round impinging jet with a nozzle-to-wall distance $H<4 D_{e}$. As for the lobed jets, the maximum value was observed in the $\mathrm{CO} / \mathrm{P}$ nozzle jet at $r / D_{\mathrm{e}}=0.58$ and $r / D_{\mathrm{e}}=0.53$ in the $\mathrm{MP}$ and $\mathrm{mP}$, respectively, and at $r / D_{\mathrm{e}}=0.19$ in both the MP and $\mathrm{mP}$ in the $\mathrm{CO} / \mathrm{H}$ nozzle jet.

In the MP of the $\mathrm{CO} / \mathrm{H}$ nozzle jet (Fig. $11 \mathrm{~b}$ ), a secondary peak appeared around $r / D_{e}=1.64$ due to the impingement of the outer sub-layer as evidenced previously in Fig. 6. Note that in Fig. 6 all 
peak locations are indicated by red ticks. The maximum value $\gamma_{\max }$ of the wall shear rate was around $9850 \mathrm{~s}^{-1}, 18000 \mathrm{~s}^{-1}$ and $28000 \mathrm{~s}^{-1}$ for the CONV, CO/P and CO/H nozzle jets, respectively (Fig. 11). For the same exit bulk-velocity $W_{b}$, the maximum wall shear rates in the $\mathrm{CO} / \mathrm{P}$ and $\mathrm{CO} / \mathrm{H}$ nozzle jets were almost two and three times higher, respectively, than that of the reference CONV nozzle jet. As the transfer mechanisms between the jet and the target wall are closely linked to the level of local skin-friction [38], the $\mathrm{CO} / \mathrm{H}$ nozzle jet would appear to be an excellent candidate for passive strategies to enhance local heat and mass transfer in industrial applications.

Below, a linkage is proposed between wall shear rate distribution and the features of the free jet region. Fig. 12 displays the profile of the mean axial velocity in the vicinity of the target wall (at $Z=1.9 D_{e}$ or $\left.Z^{*}=0.1 D_{e}\right)$ and the subsequent wall-shear rate distribution, together with the corresponding fluctuations.
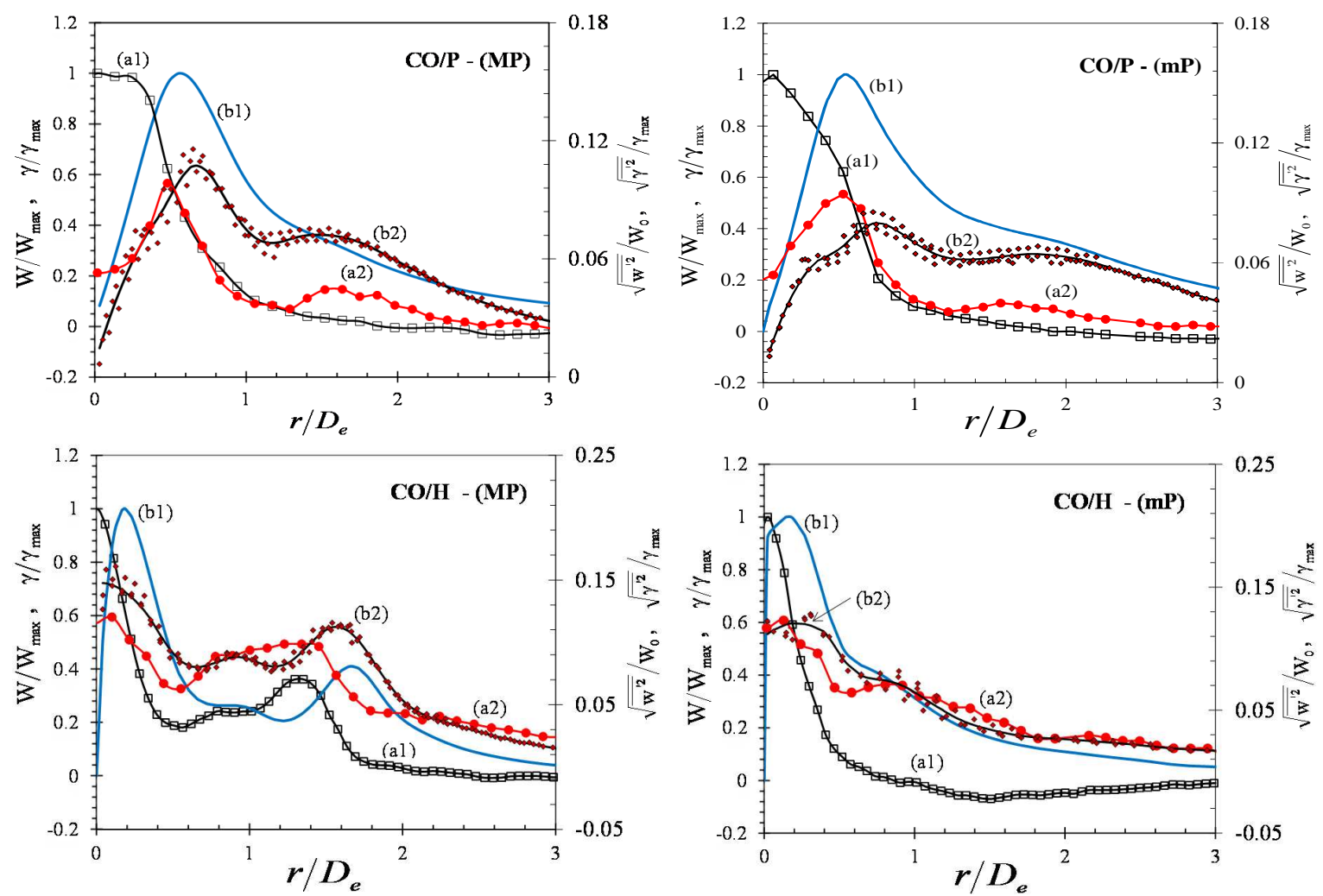

Fig.12 Mean values and fluctuations of axial velocity at $\mathrm{Z}=1.9 \mathrm{D}_{\mathrm{e}}$ and wall-shear rate of the $\mathrm{CO} / \mathrm{P}$ and $\mathrm{CO} / \mathrm{H}$ nozzle jets : (a1) $-\mathrm{W} / \mathrm{W}_{\max } ;(\mathrm{a} 2)-\sqrt{\overline{\mathrm{w}^{\prime 2}}} / \mathrm{W}_{0} ;(\mathrm{b} 1)-\gamma / \gamma_{\max } ;(\mathrm{b} 2)-\sqrt{\overline{\gamma^{\prime 2}}} / \gamma_{\max }$. The $\gamma$ profiles are the best fit curves shown in Fig.11 
As expected, the wall shear rate distributions and the free jet configuration before impingement were linked. For all three jets, the first peak of the wall shear rate was shifted slightly relative to the inflection point of the mean axial velocity. The inflection point of the mean axial velocity in the potential core of the jet corresponds to the location of the development of Kelvin Helmholtz (K-H) structures. The observed offset between this inflection point and the $\gamma_{\max }$ - position can be explained by the increase in growth and stretching of the K-H vortices when they impinged the target wall. The secondary $\gamma$ peaks in the cases where they were present, were linked to similar peaks in the mean axial velocity profile before impingement. This is clearly visible in the major plane of the $\mathrm{CO} / \mathrm{H}$ nozzle jet.

In an analogous manner to the similarity of the mean values, the rms values of the wall shear rate increased with increasing rms of axial mean velocity. The two distributions exhibit local maximum around the locations of the mean wall shear rate peaks. A similar observation was made by Roux

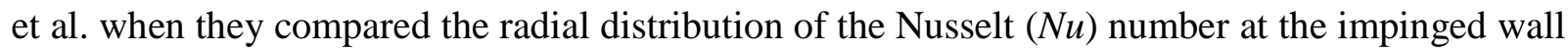
and the turbulence intensity of the axial velocity in the vicinity of the wall [20].

Hence, the peaks in $\gamma$-distribution or in $\mathrm{Nu}$-distribution matched with the high turbulence at the end of the free jet region. It is reported in the literature that the first peak in $\gamma$-distribution or in $\mathrm{Nu}$ - distribution is related to the impingement of the K-H vortices on the wall [23, 35], whereas the secondary peak is due to secondary counter-rotating vortices and to the associated turbulence produced $[37,41]$, or to a transition from a laminar to a turbulent boundary layer $[20,36]$. In the context of the present study, the first and second peaks of $\gamma$-distribution in the major plane of the $\mathrm{CO} / \mathrm{H}$ nozzle jet are related to the persistent double shear layer in this plane (Fig. $6 \mathrm{c}$ ) and the subsequent development of the vortices [54] in the shear layers.

The radial distribution of the local Sherwood number of the Cross-Shaped nozzle jets are compared in Fig. 13 to that of the convergent nozzle jet. In both the CO/P and CONV nozzles, the local Sherwood number begins to increase from the stagnation point toward a local first peak located near the nozzle edge. This peak appears in the same region where the wall shear rate is at maximum. As was mentioned above, this region corresponds to the development region of the Kelvin Helmholtz vortices. After this point, the local Sherwood number attains a plateau or a slight second peak before decreasing gradually into the wall jet region. The local Sherwood number along the major and minor planes of the $\mathrm{CO} / \mathrm{H}$ nozzle jet shows different trend. The maximum local 
Sherwood number occurs around the stagnation point and decreases monotonically. The mass transfer is at maximum at the stagnation point, probably because of higher turbulence intensity and higher wall shear rate at this point (Fig. $8 \mathrm{~b}$ and $11 \mathrm{~b}$ ). As for $\gamma$-distribution (Fig. $11 \mathrm{~b}$ ), Sh distribution (Fig. 13 b) exhibits an important second peak in the major plane of $\mathrm{CO} / \mathrm{H}$ nozzle jet, related to the presence of an outer sub-layer in this plane (Fig. $6 \mathrm{c}$, left). Hence, the wall shear rate and the local mass transfer are both nozzle geometry dependent. The maximum mass transfer for the $\mathrm{CO} / \mathrm{P}$ and $\mathrm{CO} / \mathrm{H}$ nozzles is up to $34 \%$ and $130 \%$, respectively higher than that of the CONV nozzle. The present result emphasizes that the use of a cross-shaped orifice not only increases wall shear rate, but also improves local mass transfer, and the hemispherical shape of the nozzle significantly improves the outcome.

By looking at the stagnation point, an improvement of mass transfer can be also identified as function of the nozzle exit geometry. The stagnation Sherwood number is equal to 900, 1245 and 2400 for the CONV, RO/H and RO/P nozzle jet, respectively. As described in Kristiawan et al. [44] and Meslem et al. [48], the stagnation Sherwood number can be also obtained from wall shear rate distribution using the hydrodynamic parameter $A$ (Eq. 4). In this equation, the wall shear rate $\gamma$ is a linear function of the radial distance $r$. When the wall shear rate was fitted with a linear dependence law in the stagnation region, the slope of the resulting curve is the hydrodynamic parameter $A$. The values of the stagnation Sherwood number calculated from the hydrodynamic parameter $A$ using Eq. 5 is equal to 897 and 1210 for the CONV and CO/P nozzle jet, respectively. The agreement between these values and the direct measured values is very satisfactory. The stagnation Sherwood number based on the hydrodynamic parameter $A$ is not calculated for the $\mathrm{CO} / \mathrm{H}$ nozzle jet due to the limited spatial resolution of measurements around the stagnation point. $\gamma=\partial u /\left.\partial z\right|_{z=0}=A \times r$

$S h=\frac{k D_{e}}{D_{C}}=\frac{1}{3^{1 / 3} \Gamma(4 / 3)}\left(\frac{A}{D_{C}}\right)^{1 / 3} D_{e}$

Chin and Tsang [34] proposed a correlation (Eq. 6) to approach the stagnation Sherwood number in a round impinging jet having a Reynolds number ranging from 4000 to 16000 and a nozzle to plate distance $H / D$ ranging from 0.2 to 6 . 
Using this correlation in our experiment conditions $\left(R e=5620, S c=1420, g(S c)=0.992, H / D_{e}=2\right)$ we obtain a stagnation Sherwood number of 898 which is extremely close to the value (900) obtained by direct measurement in the case of the reference round jet. Such a comparison provides confidence in the quality of our measurements.

(a)

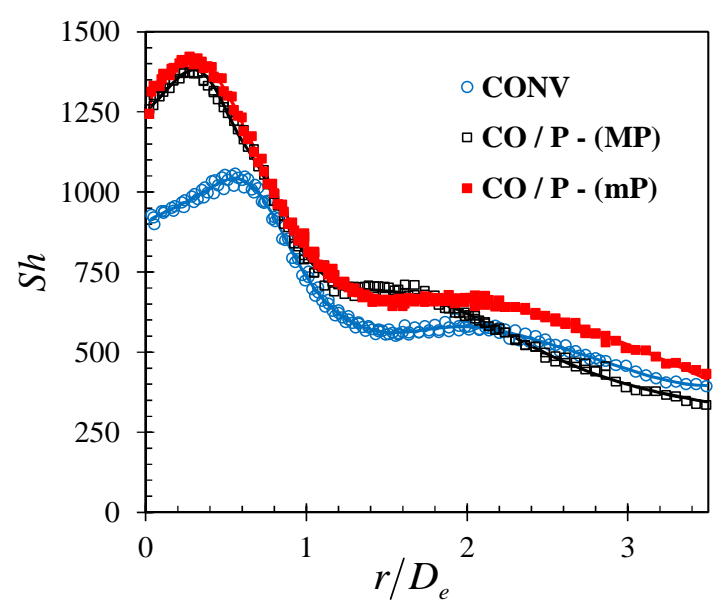

(b)

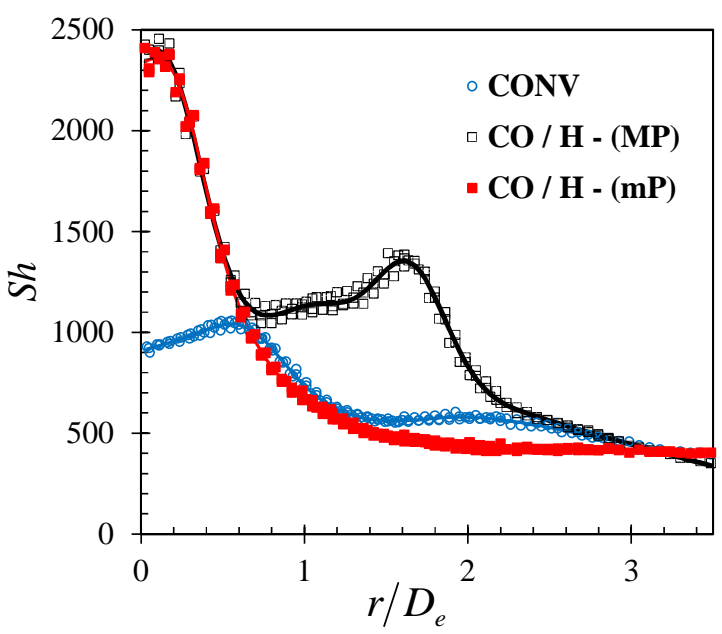

Fig.13 Sherwood number as a function of radial distance from the stagnation point: (a) CONV and $\mathrm{CO} / \mathrm{P}$ nozzles; (b) CONV and $\mathrm{CO} / \mathrm{H}$ nozzles - the solid lines are the best fit curves for the experimental data.

For the three nozzles, the global Sherwood number is obtained from the measured limiting diffusion current through the platinum disc of $3.2 D_{e}$ centred on the stagnation point (Figure $1 \mathrm{~b}$ ).

The obtained values are 495, 620 and 605 for the $\mathrm{CONV}, \mathrm{CO} / \mathrm{P}$ and $\mathrm{CO} / \mathrm{H}$ nozzle jet, respectively. Thus $\mathrm{CO} / \mathrm{P}$ nozzle jet and $\mathrm{CO} / \mathrm{H}$ nozzle jet lead to global mass transfer improvement by $25 \%$ and $22 \%$ respectively, relative to the reference CONV nozzle jet. As can be seen on Figure 13, global mass transfer improvement is mainly related to the contribution of the impingement region of the jet where impinged fluid has a higher speed. Contrary to what one can be expected, the $\mathrm{CO} / \mathrm{H}$ nozzle jet has globally the same efficiency as the CO/P nozzle jet for the considered Reynolds number, nozzle to plate distance and integration area. In our opinion, the obtained result on global mass transfer is closely dependent on the integration area limited herein to a platinum disc of $3.2 D_{e}$. 
A larger platinum disc of $12 D_{e}$ will be manufactured for new global mass transfer measurements. Anyway, in the considered conditions the cross-shaped orifice enhances the mass transfer not only locally but also globally. If a higher local or singular point mass transfer is required, the $\mathrm{CO} / \mathrm{H}$ nozzle seems to be more interesting for this aim.

\section{Conclusions}

The present study confirmed the relation between nozzle geometry and mass transfer phenomenon induced by a submerged jet impinged orthogonally on a flat plate. To generate the jet flow, three different nozzles were examined. Two Cross-Shaped orifice jets from a planar orifice nozzle $(\mathrm{CO} / \mathrm{P})$ and from a hemispherical orifice nozzle $(\mathrm{CO} / \mathrm{H})$ were compared to a reference convergent jet. The nozzle-to-wall distance was equal to 2 equivalent nozzle diameters and the Reynolds number, based on free nozzle area and on exit bulk-velocity, was 5620 in each flow. Hence in all the cases, the volumetric flow rate was conserved, and this choice is related to the aimed HVAC application. For others applications where the energy conservation is required, further investigations should be conducted at the same power input.

By combining Particle Image Velocimetry (PIV) and electrodiffusion technique (ED), mean and turbulent velocity along with wall shear rates and mass transfer were analyzed.

The switching-over phenomenon observed in the jet from the CO/P nozzle did not occur in the jet from the $\mathrm{CO} / \mathrm{H}$ nozzle. In other words, introducing a curvature to the surface containing a lobed orifice completely changed the behaviour of the jet flow generated. The curvature of the lobes in the $\mathrm{CO} / \mathrm{H}$ nozzle resulted in the conservation of the jet asymmetry. In the free jet region, a twostep distribution was conserved in the mean profiles in the major plane of the $\mathrm{CO} / \mathrm{H}$ nozzle jet, leading to a double-peak in wall shear rate and Sherwood number distributions. The CO/H nozzle jet was characterized by high axial turbulence intensity. In this jet, the maximum wall shear rate was almost two and three times higher than in the CO/P and CONV nozzle jets, respectively.

It was shown that for the same volumetric flow rate, the use of lobed nozzle not only improves wall shear rate, but also increases local and global mass transfer, which suggests a close relationship between the two quantities. The maximum local Sherwood number is $34 \%$ and $130 \%$ higher for $\mathrm{CO} / \mathrm{P}$ and $\mathrm{CO} / \mathrm{H}$ nozzles compare to the CONV nozzle. The average Sherwood number obtained over a disc of $3.2 D_{\mathrm{e}}$ in diameter reveals an improvement up to $25 \%$ and $22 \%$ for $\mathrm{CO} / \mathrm{P}$ and $\mathrm{CO} / \mathrm{H}$ 
nozzles, respectively, relatively to CONV nozzle. Hence, the $\mathrm{CO} / \mathrm{H}$ nozzle jet appears to be an excellent candidate for passive strategies to enhance both local and global mass transfer.

\section{Acknowledgments}

This work was supported by the Grants from the French National Agency of Research, the "FLUBAT" project and ANR-12-VBDU-0010

\section{References}

1. Xu, G. and R.A. Antonia, Effect of different initial conditions on a turbulent round free jet. Experiments in Fluids, 2002. 33: p. 677-683.

2. Romano, G.P., The effect of boundary conditions by the side of the nozzle of a low Reynolds number jet. Experiments in Fluids, 2002. 33: p. 323-333.

3. Quinn, W.R., Upstream nozzle shaping effects on near field flow in round turbulent free jets. European Journal of Mechanics B-Fluids, 2006. 25: p. 279-301.

4. Mi, J., G.J. Nathan, and D.S. Nobes, Mixing characteristics of axisymmetric free jets from a contoured nozzle an orifice plate and a pipe. Journal of Fluids Engineering, 2001. 123.

5. Husain, Z.D. and A.K.M.F. Hussain, Axisymetric Mixing Layer: Influence of the Initial and Boundary Conditions. AIAA Journal, 1979. 17(1): p. 48-55.

6. $\quad$ Nathan, G.J., et al., Impacts of a jet's exit flow pattern on mixing and combustion performance. Progress in Energy and Combustion Science. Progress in Energy and Combustion Science, 2006. 32(5-6): p. 496-538.

7. Hussain, A.K.M.F., Coherent structures and studies of perturbed and unperturbed jets, In The Role of Coherent Structures in Modelling Turbulence and Mixing, J. Jimenez, Editor. 1981, Springer Berlin Heidelberg. p. 252-291.

8. Hussain, A.K.M.F. and K.B.M.Q. Zaman, The 'preferred mode' of the axisymetric jet. Journal of Fluid Mechanics, 1981. 110: p. 39-71.

9. Hussain, F. and H.S. Husain, Elliptic jets. Part1. Characteristics of unexcited and excited jets. Journal of Fluid Mechanics, 1989. 208: p. 257-320.

10. Zaman, K.B.M.Q., M.F. Reeder, and M. Samimy, Control of axisymmetric jet using vortex generators. Physics of Fluids, 1994. 6(2): p. 778-793. 
11. Gutmark, E.J. and F.F. Grinstein, Flow Control with Noncircular Jets. Annual Reviews of Fluid Mechanics, 1999. 31: p. 239-272.

12. $\mathrm{Mi}$, J. and G.J. Nathan, Effect of small vortex-generators on scalar mixing in the developing region of a turbulent jet. international Journal of Heat and Mass Transfer, 1999. 42: p. 3919 3926.

13. Zaman, K.B.M.Q., F.Y. Wang, and N.J. Georgiadis, Noise, Turbulence and Thrust of Subsonic Free Jets from Lobed Nozzles. AIAA Journal, 2003. 41(3).

14. Gardon, R., Heat Transfer Between a Flat Plate and Jets of Air Impinging on It. Int. Dev. Heat Transfer (ASME), 1962: p. 454-460.

15. Gardon, R. and J.C. Akfirat, The role of turbulence in determining the heat-transfer characteristics of impinging jets. International Journal of Heat and Mass Transfer, 1965. 8: p. 1261-1272.

16. Gardon, R. and J.C. Akfirat, Heat Transfer Characteristics of Impinging Two-Dimensional Air Jets. Journal of Heat Transfer, 1966. 88(1): p. 101-107.

17. Popiel, C.O. and L. Boguslawski. Effect of Flow Structure on the heat or mass transfer on a Flat plate in Impinging Round jet. In 2nd UK National Conf. on Heat Transfer. 1988.

18. Vejrazka, J., et al., Effect of an external excitation on the flow structure in a circular impinging jet. Physics of Fluids (1994-present), 2005. 17(10): p. - .

19. Alekseenko, S.V., et al., Experimental study of an impinging jet with different swirl rates. International Journal of Heat and Fluid Flow, 2007. 28(6): p. 1340-1359.

20. Roux, S., et al., Experimental investigation of the flow and heat transfer of an impinging jet under acoustic excitation. International Journal of Heat and Mass Transfer, 2011. 54: p. 3277-3290.

21. Trávníček, Z., et al., Axisymmetric impinging jet excited by a synthetic jet system. international Journal of Heat and Mass Transfer, 2012. 55(4): p. 1279-1290.

22. Sang-Joon, L., L. Jung-Ho, and L. Dae-Hee, Local heat transfer measurements from an elliptic jet impinging on a flat plate using liquid crystal. international Journal of Heat and Mass Transfer, 1994. 37(6): p. 967-976.

23. Lee, J. and S.J. Lee, The effect of nozzle configuration on stagnation region heat transfer enhancement of axisymmetric jet impingement. International Journal of Heat and Mass Transfer, 2000. 43: p. 3497-3509.

24. Gao, N., H. Sun, and D. Ewing, Heat transfer to impinging round jets with triangular tabs. international Journal of Heat and Mass Transfer, 2003. 46(14): p. 2557-2569.

25. Nakod, P.M., S.V. Prabhu, and R.P. Vedula, Heat transfer augmentation between impinging circular air jet and flat plate using finned surfaces and vortex generators. Experimental Thermal and Fluid Science, 2008. 32(5): p. 1168-1187.

26. Violato, D., et al., Three-dimensional vortex dynamics and convective heat transfer in circular and chevron impinging jets. International Journal of Heat and Fluid Flow, 2012. 37: p. 22-36.

27. Herrero Martin, R. and J.M. Buchlin, Jet impingement heat transfer from lobed nozzles. International Journal of Thermal Sciences, 2011. 50(7): p. 1199-1206.

28. Gulati, P., V. Katt, and S.V. Prabhu, Influence of the shape of the nozzle on local heat transfer distribution between smooth flat surface and impinging air jet. International Journal of Thermal Sciences, 2009. 48: p. 602-617.

29. Gutmark, E.J. and C.M. Ho, Preferred modes and the spreading rates of jets. Physics of Fluids, 1983. 26(10): p. 2932-2938.

30. Nastase, I. and A. Meslem, Vortex dynamics and mass entrainment in turbulent lobed jets with and without lobe deflection angles. Experiments in Fluids, 2010. 48(4): p. 693-714.

31. Hu, H., et al., Particle Image Velocimetry and Planar Laser Induced Fluorescence Measurements on Lobed Jet Mixing Flows. Experiments in Fluids (Suppl.), 2000: p. S141-S157.

32. Vallis, E.A., M.A. Patrick, and A.A. Wragg. Techniques of wall measurements in fluid mechanics. In Euromech.90. 1977. Nancy, France. 
33. Kataoka, K., et al., Mass transfer between a plane surface and an impinging turbulent jet: the influence of surface-pressure fluctuations. Journal of Fluid Mechanics, 1982. 119: p. 91-105.

34. Chin, D.T. and C.H. Tsang, Mass Transfer to an Impinging Jet Electrode. Journal of The Electrochemical Society, 1978. 125(9): p. 1461-1470.

35. Kataoka, K. and T. Mizushina. Local enhancement of the rate of heat-transfer in an impinging round jet by free-stream turbulence. In Heat Transfer 1974; Proceedings of the Fifth International Conference, Tokyo, Volume 2. 1974. Tokyo.

36. Lytle, D. and B.W. Webb, Air jet impingement heat transfer at low nozzle-plate spacings. International Journal of Heat and Mass Transfer, 1994. 37(12): p. 1687-1697.

37. Hadziabdic, M. and K. Hanjalic, Vortical structures and heat transfer in a round impinging jet. Journal of Fluid Mechanics, 2008. 596: p. 221-260.

38. Alekseenko, S.V. and D.M. Markovich, Electrodiffusion diagnostics of wall shear stresses in impinging jet. Journal of Applied Electrochemistry, 1994. 24: p. 626-631.

39. Phares, D.J., G.T. Smedley, and R.C. Flagan, The wall shear stress produced by the normal impingement of a jet on a flat surface. Journal of Fluid Mechanics, 2000. 418: p. 351-375.

40. Tummers, M.J., J. Jacobse, and S.G.J. Voorbrood, Turbulent flow in the near field of a round impinging jet. international Journal of Heat and Mass Transfer, 2011. 54: p. 4939-4948.

41. El-Hassan, M., et al., Experimental investigation of the wall shear stress and the vortex dynamics in a circular impinging jet. Experiments in Fluids, 2012. 52(6): p. 1475-1489.

42. Hall, J.W. and D. Ewing, On the dynamics of the large-scale structures in round impinging jets. Journal of Fluid Mechanics, 2006. 555: p. 439-458.

43. El-Hassan, M. and A. Meslem, Time-resolved stereoscopic PIV investigation of the entrainement in the near-field of circular and daisy-shaped orifice jets. Physics of Fluids, 2010. 22(035107): p. $26 \mathrm{p}$.

44. Kristiawan, M., et al., Wall shear rates and mass transfer in impinging jets: Comparison of circular convergent and cross-shaped orifice nozzles. International Journal of Heat and Mass Transfer, 2012. 55: p. 282-293.

45. Rajaratnam, N., Turbulent jets. 1976, Amsterdam, Netherlands: Elsevier Scientific Publishing Company.

46. Belovich, V.M. and M. Samimy, Mixing processes in a coaxial geometry with a central lobed mixer-nozzle. AIAA Journal, 1997. 35(5).

47. Bolashikov, Z., et al., Improved inhaled air quality at reduced ventilation rate by control of airflow interaction at the breathing zone with lobed jets. HVAC\&R Research, 2013. 20(2): p. 238-250.

48. Meslem, A., et al., Flow dynamics and mass transfer in impinging circular jet at low Reynolds number. Comparison of convergent and orifice nozzles. International Journal of Heat and Mass Transfer, 2013. 67(0): p. 25-45.

49. Scarano, F., L. Riethmuller M, and J. Adrian R, Advances in iterative multigrid PIV image processing. Experiments in Fluids, 2000. 29(3): p. S51-S60.

50. Westerweel, J., Theoretical analysis of the measurement precision in particle image velocimetry. Experiments in Fluids, 2000. 29(1): p. S003-S012.

51. Reiss, L.P. and T.J. Hanratty, Measurement of instantaneous rates of mass transfer to a small sink on a wall. AIChE Journal, 1962. 8(2): p. 245-247.

52. Nastase, I., A. Meslem, and P. Gervais, Primary and secondary vortical structures contribution in the entrainement of low Reynolds number jet flows. Experiments in Fluids, 2008. 44(6): p. 10271033.

53. Hu, H., et al., Research on the Vortical and Turbulent Structures in the Lobed jet Flow Using Laser Induced Fluorescence and Particle Image Velocimetry Techniques. Measurement Science and Technology, 2000. 11: p. 698-711.

54. Nastase, I., A. Meslem, and M. El-Hassan, Image processing analysis of vortex dynamics of lobed jets from three-dimensional diffusers. Fluid Dynamics Research, 2011. 43(6): p. 065502. 
55. El-Hassan, M., A. Meslem, and K. Abed-Meraïm, Experimental investigation of the flow in the near-field of a cross-shaped orifice jet. Phys. Fluids, 2011. 23(045101): p. 16 p.

56. Nastase, I., et al., Lobed grilles for high mixing ventilation - An experimental analysis in a full scale model room. Building and Environment, 2011. 46(3): p. 547-555.

57. Gorman, J.M., E.M. Sparrow, and J.P. Abraham, Slot jet impingement heat transfer in the presence of jet-axis switching. International Journal of Heat and Mass Transfer, 2014. 78(0): p. 50-57.

58. Baydar, E. and Y. Ozmen, An experimental investigation on flow structures of confined and unconfined impinging air jets. Heat and Mass Transfer, 2006. 42(4): p. 338-346.

59. Todde, V., P.G. Spazzini, and M. Sandberg, Experimental analysis of low-Reynolds number free jets. Evolution along the jet centerline and Reynolds number effects. Experiments in Fluids, 2009. 47: p. 279-294.

60. Cooper, D., et al., Impinging jet studies for turbulence model assessment-I. Flow-field experiments. international Journal of Heat and Mass Transfer, 1993. 36(10): p. 2675-2684.

61. Kazuya, I., et al., Heat transfer characteristics of a planar water jet impinging normally or obliquely on a flat surface at relatively low Reynolds numbers. Experimental thermal and fluid science, 2009. 33(8): p. 1226-1234.

62. Glauert, M.B., The wall jet. Journal of Fluid Mechanics, 1956. 1(06): p. 625-643.

63. Tummers, M.J., J. Jacobse, and S.G.J. Voorbrood, Turbulent flow in the near field of a round impinging jet. international Journal of Heat and Mass Transfer, 2011. 54(23-24): p. 4939-4948.

64. Xu, Z. and H. Hangan, Scale, boundary and inlet condition effects on impinging jets. Journal of Wind Engineering and Industrial Aerodynamics, 2008. 96(12): p. 2383-2402. 